\title{
Field quantization, photons and non-Hermitean modes.
}

\author{
S. A. Brown ${ }^{(a)}$ and B. J. Dalton ${ }^{(a),(b)}$ \\ (a) Department of Physics, University of Queensland, St Lucia, \\ Queensland 4072, Australia \\ (b) Sussex Centre for Optical and Atomic Physics, \\ University of Sussex, Brighton BN1 9QH, United Kingdom
}

October 24, 2018

\begin{abstract}
Field quantization in unstable optical systems is treated by expanding the vector potential in terms of non-Hermitean (Fox-Li) modes. We define non-Hermitean modes and their adjoints in both the cavity and external regions and make use of the important bi-orthogonality relationships that exist within each mode set. We employ a standard canonical quantization procedure involving the introduction of generalised coordinates and momenta for the electromagnetic (EM) field. Three dimensional systems are treated, making use of the paraxial and monochromaticity approximations for the cavity non-Hermitean modes. We show that the quantum EM field is equivalent to a set of quantum harmonic oscillators (QHO), associated with either the cavity or the external region non-Hermitean modes, and thus confirming the validity of the photon model in unstable optical systems. Unlike in the conventional (Hermitean mode) case, the annihilation and creation operators we define for each QHO are not Hermitean adjoints. It is shown that the quantum Hamiltonian for the EM field is the sum of non-commuting cavity and external region contributions, each of which can be expressed as a sum of independent QHO Hamiltonians for each non-Hermitean mode, except that the external field Hamiltonian also includes a coupling term responsible for external nonHermitean mode photon exchange processes. The non-commutativity of certain cavity and external region annihilation and creation operators is associated with cavity energy gain and loss processes, and may be described in terms of surface integrals involving cavity and external region non-Hermitean mode functions on the cavity-external region boundary. Using the essential states approach and the rotating wave approximation, our results are applied to the spontaneous decay of a two-level atom inside an unstable cavity. We find that atomic transitions leading to cavity non-Hermitean mode photon absorption are associated with a different coupling constant to that for transitions leading to photon emission, a feature consequent on the use of non-Hermitean mode functions. We show
\end{abstract}


that under certain conditions the spontaneous decay rate is enhanced by the Petermann factor.

\section{Introduction}

The photon model of the quantum electromagnetic (EM) field has recently been developed further for the areas of cavity QED and quantum optics in dielectric media. Recent work has demonstrated its validity in microscopic theories [1], where EM field quantization is the same as in free space, and in macroscopic theories, where the classical optical device or material medium is treated as a spatially dependent permittivity. Canonical quantization carried out by expanding the vector potential in terms of the true (or universe) modes for the system (obtained from a Helmholtz equation) shows that the EM field is again equivalent to uncoupled quantum harmonic oscillators (QHO), one for each true

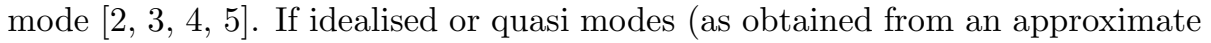
optical system permittivity) are used instead of true modes, coupled QHO are obtained, one for each quasi mode [3, 6] and photon exchange processes between quasi modes occur. Phenomena such as beam splitter effects [7], light energy loss from cavities [8], reflection and refraction [9] can be explained via such processes using quasi mode theory. Both localised quasi mode (as in cavity or external regions for the standard cavity QED model [§]) and non-localised quasi mode (as in the beam splitter [7]) cases occur. Quasi mode theory has been extended [10] to cases [9] where two sets of quasi modes are needed.

The photon model obtained by quantization based on normal mode (free space, true or quasi mode) expansions involves mode functions determined from Hermitean eigenvalue equations and which satisfy standard orthogonality conditions (power orthogonality). However, for unstable optical resonators or gainguided lasers, the natural EM field modes to use (Fox-Li modes) are eigenfunctions of a non-Hermitian operator. Biorthogonality conditions apply for these non-Hermitean modes (NHM) and the related set of Hermitean adjoint mode functions (HAM). The appropriateness of the photon model [11] has been questioned for such systems, where phenomena such as linewidth enhancement in gain-guided lasers occur. This linewidth enhancement was interpreted [12] as excess spontaneous emission into the laser mode and described by the Petermann factor, and several semiclassical studies (for example 13, 14, 15]) have successfully accounted for the experimentally observed Peterman factors using the normalisation integrals for the HAM functions (the original NHM are normalised to unity). A variety of differing quantum treatments aimed at explaining the linewidth enhancement have been published. Some have the objective of trying to account for the success of the semiclassical theories in terms of a fully quantum treatment of the field, others present other explanations of the excess noise without using the NHM functions. In [16] the enhanced linewidth is attributed as a propagation effect arising from longitudinally inhomogeneous noise gain, rather than an excess of local spontaneous emission, the microscopic rate of spontaneous emission into a given non-power-orthogonal cavity mode 
not being enhanced by the Petermann factor. Reference [17] accounts for the excess noise factor via either spontaneous emission noise or amplification of vacuum modes leaking into the cavity, depending on the choice made for operator ordering. This treatment did not involve the use of NHM and single atom decay was found to be enhanced by the cavity $\mathrm{Q}$ factor, not the Petermann factor. In [18] a simple few mode model was presented, and excess noise attributed to loss-induced coupling between the cavity eigenmodes, with no use of NHM being made. In reference [19] on the other hand, field quantization based on the NHM and the HAM is described, and the spontaneous emission rate of an atom in the cavity found to be enhanced by the Petermann factor. The work in 20] is a laser theory involving a reservoir of excited atoms. Starting from a true mode description, quasimodes and their adjoints satisfying non-Hermitean eigenvalue equations are introduced, and results approximating to those for the semiclassical theories are obtained for the noise amplification. Finally, in reference [21] field quantization based on NHM and the HAM obtained by solving the Helmholtz equation in both the cavity and external regions with non-Hermitean boundary conditions is presented. As in 17] the single atom decay was found to be enhanced by the cavity $\mathrm{Q}$ factor, not the Petermann factor.

The present paper is a further quantum treatment involving a standard canonical quantization proceedure based on expanding the vector potential in the unstable cavity region via the non-Hermitean (Fox-Li) modes and their Hermitean adjoint modes. It is aimed at trying to account for the success of the semiclassical theories in terms of a fully quantum treatment of the field. Our approach is similar to those of [19, 21, although here we employ the canonical quantization method and also treat three dimensional systems, using the paraxial [22] and monochromaticity approximations [19] for the cavity NHM. As in [19], 21], we consider all field modes, rather than a small number as in the simplified model treated in [18]. Our NHM and HAM are also obtained from the properties of the unstable optical system, rather than from treating atom-field interactions, as in [20]. As in [21], the external region is treated and the field described via further NHM and HAM. Similarly to [19], [21], we find that the field is equivalent to a set of QHO's, associated now with nonHermitean modes rather than true modes and thus confirming the validity of the photon model for the case of unstable optical systems. Similarly to [19], [21], the annihilation, creation operator pairs for each QHO are not Hermitean adjoints. However, our results differ significantly in detail from those in [19], [21]. Our canonical quantization procedure involving both right and left travelling modes leads to a doubling of the annihilation, creation operator pairs compared to [19], 21. We show that the total number of true mode QHO's equals the total number of QHO's associated with either with the cavity or the external region NHM, one oscillator for each annihilation, creation operator pair. As in [21], the final form of our quantum Hamiltonian for the EM field is the sum of non-commuting cavity and external region contributions, but is simpler as there are no off-diagonal terms. To a good approximation, both the cavity and external region Hamiltonians can be expressed as the sum of independent QHO Hamiltonians for each NHM, but the external region Hamiltonian also includes 
a coupling term responsible for external NHM photon exchange processes. The two independent QHO Hamiltonians are alternative choices for an unperturbed Hamiltonian. Analogous to [19] for the cavity region Hamiltonian, we obtain left and right eigenstates for each of these unperturbed Hamiltonians and the energy is given by the usual QHO result. As in [21], certain cavity NHM and external region NHM annihilation, creation operators do not commute, leading to cavity energy gain and loss processes, though again the details differ. We find a simple description of the resultant cavity-external region light coupling in terms of surface integrals involving products of cavity and external NHM functions. Atom-field interactions are treated in the electric dipole and rotating wave approximations. We show that atomic transitions leading to cavity NHM photon absorption are associated with a different coupling constant to that for atomic transitions leading to photon emission. This feature is directly related to the treatment being based on NHM functions and leads to enhanced emission rates. Using the essential states approach, we consider spontaneous decay of a two level atom located in the cavity. The coupling term in the field Hamiltonian is neglected, assuming that atomic decay into the cavity is much faster than cavity decay. Coupled equations are obtained for atom-field states amplitudes involving the excited atom and no photons or the ground state atom with one photon in a cavity NHM. Markovian decay occurs under certain conditions, the decay rate being enhanced by the Petermann factor 12 in special cases when a single NHM dominates.

The plan of this paper is as follows. In Section 2 quantization of the EM field based on NHM is carried out and approximate energy eigenstates determined. In Section 3 atom-field interactions are considered and a simple treatment of spontaneous emission from a two level atom into an unstable optical cavity is presented. The results are summarised in Section 1 . The Appendix contains the detailed calculation of certain commutation rules.

\section{Theory of canonical quantization via non-Hermitean modes}

\section{$2.1 \quad$ Non-Hermitean mode functions}

The case of interest is that of unstable optical cavities, for which a typical example is shown in figure 1. Using the paraxial approximation [22], 23, 24], the cavity NHM functions $\mathbf{U}_{n}(\mathbf{R})$ and HAM functions $\mathbf{V}_{n}(\mathbf{R})$ describing right propagating fields associated with light of angular frequency $\omega_{n}$ can be written:

$$
\begin{aligned}
& \mathbf{U}_{n}(\mathbf{R})=\mathcal{N} \hat{\boldsymbol{\alpha}}_{n} \cdot \exp \left(i k_{n} z\right) \cdot u_{n}(\mathbf{s}, z) \\
& \mathbf{V}_{n}(\mathbf{R})=\mathcal{N} \hat{\boldsymbol{\alpha}}_{n} \cdot \exp \left(i k_{n} z\right) \cdot v_{n}(\mathbf{s}, z)
\end{aligned}
$$

with the polarization vectors chosen as $\hat{\boldsymbol{\alpha}}_{n}=(\hat{\mathbf{x}}$ or $\hat{\mathbf{y}})$ and the wave number is $k_{n}=\omega_{n} / c(\geq 0) . \mathcal{N}$ is a normalization factor. The fields $u_{n}(\mathbf{s}, z)$ and $v_{n}(\mathbf{s}, z)$ are slowly varying functions of $z$ describing the transverse $\mathbf{s}=(x, y)$ dependence 
of the NHM functions. These fields satisfy the right propagating paraxial wave equation:

$$
\left(\nabla_{T}^{2}+2 i k_{n} \partial / \partial z\right) u_{n}(\mathbf{s}, z)=\left(\nabla_{T}^{2}+2 i k_{n} \partial / \partial z\right) v_{n}(\mathbf{s}, z)=0,
$$

where $\boldsymbol{\nabla}_{T}=\hat{\mathbf{x}} \partial / \partial x+\hat{\mathbf{y}} \partial / \partial y$ is the transverse component of $\boldsymbol{\nabla}$. In addition, $u_{n}(\mathbf{s}, z)$ and $v_{n}(\mathbf{s}, z)$ satisfy eigenvalue equations of the form:

$$
\begin{aligned}
\hat{£} u_{n}(\mathbf{s}, z) & =\gamma_{n} u_{n}(\mathbf{s}, z) \\
\hat{£}^{\dagger} v_{n}(\mathbf{s}, z) & =\gamma_{n}^{*} v_{n}(\mathbf{s}, z),
\end{aligned}
$$

where $\hat{£}$ is a two dimensional non-Hermitean round-trip propagation operator describing right travelling light, $\hat{£}^{\dagger}$ is the related Hermitean adjoint operator

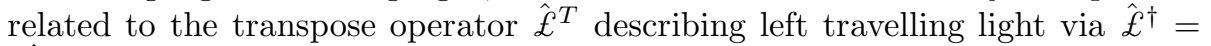
$\left(\hat{£}^{T}\right)^{*}$, and $\gamma_{n}$ are the complex eigenvalues which are independent of $z$. The


the $z$ plane into a new field in the same plane, and are defined in terms of the propagation kernel $G_{+}\left(\mathbf{s}, z ; \mathbf{s}_{0}, z\right)$ such that for any arbitary transverse function $f(\mathbf{s}, z)$ we have:

$$
\begin{aligned}
g(\mathbf{s}, z) & =\hat{£} f(\mathbf{s}, z)=\int d^{2} \mathbf{s}_{0} G_{+}\left(\mathbf{s}, z ; \mathbf{s}_{0}, z\right) f\left(\mathbf{s}_{0}, z\right) \\
h(\mathbf{s}, z) & =\hat{£}^{T} f(\mathbf{s}, z)=\int d^{2} \mathbf{s}_{0} G_{+}\left(\mathbf{s}_{0}, z ; \mathbf{s}, z\right) f\left(\mathbf{s}_{0}, z\right) \\
i(\mathbf{s}, z) & =\hat{£}^{\dagger} f(\mathbf{s}, z)=\int d^{2} \mathbf{s}_{0} G_{+}^{*}\left(\mathbf{s}_{0}, z ; \mathbf{s}, z\right) f\left(\mathbf{s}_{0}, z\right) .
\end{aligned}
$$

Thus $\hat{£}$ maps $f(\mathbf{s}, z)$ onto $g(\mathbf{s}, z), \hat{£}^{T}$ maps $f(\mathbf{s}, z)$ onto $h(\mathbf{s}, z)$ and $\hat{£}^{\dagger}$ maps $f(\mathbf{s}, z)$ onto $i(\mathbf{s}, z)$. The propagation kernel $G_{+}\left(\mathbf{s}, z ; \mathbf{s}_{0}, z\right)$ is obtained from the Fresnel approximation to the Huygens-Fresnel principle, details are given in references [23, 24]. For the situation depicted in figure 1, $G_{+}$would be constructed allowing for right travelling propagation from the $z$ plane to the right end mirror, left travelling propagation to the left end mirror and then right travelling propagation to the $z$ plane. The fields $\hat{£} f(\mathbf{s}, z)$ and $\hat{£}^{\dagger} f(\mathbf{s}, z)$ also satisfy the same paraxial wave equations as $u_{n}(\mathbf{s}, z)$ and $v_{n}(\mathbf{s}, z)$. In our notation $n$ will specify $\hat{\boldsymbol{\alpha}}_{n}, k_{n}$ (the polarization and longitudinal wave number) and the transverse mode index $\theta_{n}$ for the distinct transverse NHM functions in which the quantities $\hat{\boldsymbol{\alpha}}_{n}, k_{n}$ are the same. It will be convenient to quantize the wave numbers, with $k_{n}=N_{n} \pi / l$, where $N_{n}$ is an integer and $l$ is the cavity length. Both $\mathbf{U}_{n}, \mathbf{V}_{n}$ describe right propagating light fields, whereas

$$
\mathbf{W}_{n}=\hat{\boldsymbol{\alpha}}_{n} \cdot \exp \left(i k_{n} z\right) \cdot w_{n}(\mathbf{s}, z)=\left(\mathbf{V}_{n}\right)^{*}
$$

describes left propagating light fields, where $w_{n}(\mathbf{s}, z)=v_{n}(\mathbf{s}, z)^{*}$ and satisfies the eigenvalue equation $\hat{£}^{T} w_{n}(\mathbf{s}, z)=\gamma_{n} w_{n}(\mathbf{s}, z)$, . Thus we see that $u_{n}(\mathbf{s}, z)$ and $v_{n}(\mathbf{s}, z)$ are special solutions of the right propagating paraxial wave equation that are self-reproducing under the propagation operators $\hat{£}$ and $\hat{£}^{\dagger}$ respectively. 
Based on the well known two dimensional orthogonality results [13] for the $u_{n}$ and $v_{n}$ such as $\int d^{2} \mathbf{s} u_{n}(\mathbf{s}, z)^{*} v_{m}(\mathbf{s}, z)=\delta_{n m}$, we can obtain important three dimensional orthogonality integrals in the form:

$$
\begin{aligned}
& \int_{C} d^{3} \mathbf{R} \mathbf{U}_{n}(\mathbf{R})^{*} \bullet \mathbf{V}_{m}(\mathbf{R})=\delta_{n m} \\
& \int_{C} d^{3} \mathbf{R} \mathbf{U}_{n}(\mathbf{R})^{*} \bullet \mathbf{U}_{m}(\mathbf{R})=\mathbf{C}_{n m} \\
& \int_{C} d^{3} \mathbf{R} \mathbf{V}_{n}(\mathbf{R})^{*} \bullet \mathbf{V}_{m}(\mathbf{R})=\mathbf{D}_{n m},
\end{aligned}
$$

the first being the biorthogonality condition, the others giving the overlap integrals for the NHM and the HAM in terms of the Hermitean, positive definite transformation matrices $\mathbf{C}$ and $\mathbf{D}$. The integrals are over the cavity region. It can be shown that $\mathbf{C}_{n m}$ and $\mathbf{D}_{n m}$ are zero unless the angular frequencies $\omega_{n}, \omega_{m}$ and the polarizations $\hat{\boldsymbol{\alpha}}_{n}, \hat{\boldsymbol{\alpha}}_{m}$ are equal, and $\mathbf{C D}=\mathbf{D} \mathbf{C}=\mathbf{E}$. By convention we choose $\mathbf{C}_{n n}=1$, and then from Schwarz' inequality it follows that $\mathbf{D}_{n n} \geqslant 1$. The Petermann factor $K_{n}$ is given by $\mathbf{D}_{n n}$ in the semiclassical theories (see [13, 14, 15]). The NHM functions satisfy a completeness relation:

$$
\sum_{n}\left(U_{n}^{\alpha}(\mathbf{R}) V_{n}^{\beta}\left(\mathbf{R}^{\prime}\right)^{*}+V_{n}^{\alpha}(\mathbf{R})^{*} U_{n}^{\beta}\left(\mathbf{R}^{\prime}\right)\right)=\delta_{\alpha \beta} \delta\left(\mathbf{R}-\mathbf{R}^{\prime}\right)
$$

for $\mathbf{R}, \mathbf{R}^{\prime}$ inside the cavity, and where $U_{n}^{\alpha}(\mathbf{R}), V_{n}^{\alpha}(\mathbf{R})$ are the $\alpha$ components $(\alpha, \beta=x, y)$ of the vector fields $\mathbf{U}_{n}(\mathbf{R}), \mathbf{V}_{n}(\mathbf{R})$. Integrals similar to those in equations (10, 11, 12) but without the complex conjugation are ignored due to the product of the two fast-varying $\exp \left(i k_{n} z\right)$ factors approximately averaging to zero. The corresponding completeness relationship in reference [19] does not include the second term. There are also interrelationships between the NHM and HAM functions in terms of the transformation matrices:

$$
\mathbf{U}_{n}(\mathbf{R})=\sum_{m} \mathbf{C}_{m n} \mathbf{V}_{m}(\mathbf{R}) \quad \mathbf{V}_{n}(\mathbf{R})=\sum_{m} \mathbf{D}_{m n} \mathbf{U}_{m}(\mathbf{R})
$$

\subsection{Vector potential and generalised coordinates}

The NHM functions and true mode functions represent monochromatic fields. For more general time dependent fields we can write the vector potential $\mathbf{A}(\mathbf{R})$ as the sum of a right travelling light field $\mathbf{A}_{R}(\mathbf{R})$ and a left travelling field $\mathbf{A}_{R}(\mathbf{R})^{*}$ for $\mathbf{R}$ inside or outside the cavity:

$$
\mathbf{A}(\mathbf{R})=\mathbf{A}_{R}(\mathbf{R})+\mathbf{A}_{R}(\mathbf{R})^{*},
$$

and then expand $\mathbf{A}_{R}(\mathbf{R})$ as a linear combination of suitable monochromatic fields. One choice is to expand $\mathbf{A}_{R}$ in terms of true mode functions $\mathbf{A}_{k}(\mathbf{R})$ 
in the right half space $R$. Corresponding true modes in the left half space are denoted $\mathbf{A}_{k *}(\mathbf{R})=\left(\mathbf{A}_{k}(\mathbf{R})\right)^{*}$. These modes all have the same angular frequency $\omega_{k}$. Alternatively, a similar expansion can be made (but only within the cavity) in terms of either the NHM functions $\mathbf{U}_{n}(\mathbf{R})$ or the HAM functions $\mathbf{V}_{n}(\mathbf{R})$. The expansion coefficients ( $q_{k}$ or $Q_{n}$ or $R_{n}$ for the three choices) act as generalised coordinates specifying the vector potential in the appropriate region. In the left half space $q_{k *}=\left(q_{k}\right)^{*}$. Thus:

$$
\begin{aligned}
& \mathbf{A}_{R}(\mathbf{R})=\sum_{k}^{R} q_{k} \mathbf{A}_{k}(\mathbf{R}) \\
& \mathbf{A}_{R}(\mathbf{R})=\sum_{n} Q_{n} \mathbf{U}_{n}(\mathbf{R})=\sum_{n} R_{n} \mathbf{V}_{n}(\mathbf{R}),
\end{aligned}
$$

where $\mathbf{R}$ lies within the cavity in equations (17). The $Q_{n}, R_{n}$ depend on the $q_{k}$, as the latter specify the field everywhere via equation (16). We have:

$$
Q_{n}=\sum_{k}^{R} \Gamma_{n k} q_{k} \quad R_{n}=\sum_{k}^{R} \Lambda_{n k} q_{k}
$$

where

$$
\begin{aligned}
\Gamma_{n k} & =\int_{C} d^{3} \mathbf{R} \mathbf{V}_{n}(\mathbf{R})^{*} \cdot \mathbf{A}_{k}(\mathbf{R}) \\
\Lambda_{n k} & =\int_{C} d^{3} \mathbf{R} \mathbf{U}_{n}(\mathbf{R})^{*} \cdot \mathbf{A}_{k}(\mathbf{R}) .
\end{aligned}
$$

The matrices $\Gamma, \Lambda$ have properties $\Gamma^{\dagger} \Lambda=\Lambda^{\dagger} \Gamma=\mathbf{E}$ and $\left(\Lambda^{\dagger} \Lambda\right)_{n m}=\mathbf{C}_{n m}$, $\left(\Gamma^{\dagger} \Gamma\right)_{n m}=\mathbf{D}_{n m}$. Interrelationships between the $Q_{n}$ and the $R_{n}$ can easily be found in terms of $\mathbf{C}, \mathbf{D}$. These are:

$$
Q_{n}=\sum_{m} \mathbf{D}_{n m} R_{m} \quad R_{n}=\sum_{m} \mathbf{C}_{n m} Q_{m}
$$

Being determined from solutions of the paraxial wave equation, the cavity NHM and HAM functions $\mathbf{U}_{n}$ and $\mathbf{V}_{n}$ actually represent the lowest order terms in a more general treatment starting from paraxial fields. Following Ref. [22], the spatial part of the vector potential in the Coulomb gauge

$$
\boldsymbol{\nabla} \cdot \mathbf{A}=0
$$

for harmonic solutions of the wave equation (and which represent right travelling waves), can be expressed as

$$
\mathbf{A}_{R}^{\left(k_{n}\right)}(\mathbf{R})=\exp \left(i k_{n} z\right) \cdot\left[\mathbf{F}_{T}(\mathbf{R})+\hat{\mathbf{z}} F_{Z}(\mathbf{R})\right]
$$


where $\mathbf{F}_{T}$ and $\hat{\mathbf{z}} F_{Z}$ are transverse and longitudinal components. $\mathbf{F}_{T}$ and $F_{Z}$ can be expressed as power series in the small quantity $f=w_{0} / l_{k}$, where $w_{0}$ is the lateral size for the beam and $l_{k}=k\left(w_{0}\right)^{2}$ is the Fraunhofer diffraction length. $\mathbf{F}_{T}, F_{Z}$ involve even, odd powers of $f$ respectively. The zeroth order term in $\mathbf{F}_{T}$ satisfies the paraxial wave equation [22], thereby clarifying the status of this equation as determining the lowest order contribution to $\mathbf{A}_{R}^{\left(k_{n}\right)}$. The cavity NHM and HAM functions $\mathbf{U}_{n}$ and $\mathbf{V}_{n}$ are particular zeroth order solutions for $\mathbf{F}_{T}$. However, to satisfy the Coulomb gauge condition even in zeroth order, the first order term from $F_{Z}$ would need to be included. This would replace $\mathbf{U}_{n}$ and $\mathbf{V}_{n}$ by:

$$
\begin{aligned}
& \mathbf{U}_{n}^{(1)}=\left(\mathbf{U}_{n}+i / k_{n} \hat{\mathbf{z}}\left\{\boldsymbol{\nabla}_{T} \cdot \mathbf{U}_{n}\right\}\right) \\
& \mathbf{V}_{n}^{(1)}=\left(\mathbf{V}_{n}+i / k_{n} \hat{\mathbf{z}}\left\{\boldsymbol{\nabla}_{T} \cdot \mathbf{V}_{n}\right\}\right),
\end{aligned}
$$

resulting in the gauge condition being satisfied correct to the second order in $f$. In a higher order treatment we would replace $\mathbf{U}_{n}$ and $\mathbf{V}_{n}$ by these expressions, which now would have a small first order correction in the $\hat{\mathbf{z}}$ direction. However, it is apparent that the small correction terms are perpendicular to the zeroth order terms $\mathbf{U}_{n}$ or $\mathbf{V}_{n}$. This results in expressions to terms in the Lagrangian and Hamiltonian based on NHM expansions only changing in second order, and such corrections can be neglected in our results. Although the Coulomb gauge condition plays a central role in the field quantization presented here, the correction terms in the NHM functions need not concern us further, since our formal canonical quantization procedure is based on the true mode functions 四. These are constructed to satisfy the Coulomb gauge condition for the free space situation that applies both inside and outside the cavity.

Expansions of the right travelling field $\mathbf{A}_{R}(\mathbf{R})$ may also be made in the external region in terms of NHM $\mathbf{U}_{K}(\mathbf{R})$ and their HAM $\mathbf{V}_{K}(\mathbf{R})$ as determined from other non-Hermitean operators, and the expansion coefficients $Q_{K}, R_{K}$ act as generalised coordinates for the field in the external region. We do not need to specify the actual method for determining these mode functions other than noting that the paraxial approximation would not apply. Generalisations to three dimensions of the approach in [21] might be applied. However, the external NHM functions should satisfy the Coulomb gauge condition and the biorthogonality conditions, and be chosen so that the magnetic energy contribution to the Lagrangian is in diagonal form (see equation (40)). Orthogonality, completeness and interrelationships analogous to equations (10, 11, 12, 13, 14) occur for the $\mathbf{U}_{K}(\mathbf{R})$ and $\mathbf{V}_{K}(\mathbf{R})$. Equations analogous to equations (17, 18, 21) apply for the $Q_{K}, R_{K}$. The matrices $\mathbf{C}, \mathbf{D}, \Gamma, \Lambda$ are replaced by $\mathbf{G}, \mathbf{H}, \Delta, \Phi$, where:

$$
\begin{aligned}
& \int_{E} d^{3} \mathbf{R} \mathbf{U}_{K}(\mathbf{R})^{*} \cdot \mathbf{V}_{L}(\mathbf{R})=\delta_{K L} \\
& \int_{E} d^{3} \mathbf{R} \mathbf{U}_{K}(\mathbf{R})^{*} \cdot \mathbf{U}_{L}(\mathbf{R})=\mathbf{G}_{K L} \\
& \int_{E} d^{3} \mathbf{R} \mathbf{V}_{K}(\mathbf{R})^{*} \cdot \mathbf{V}_{L}(\mathbf{R})=\mathbf{H}_{K L}
\end{aligned}
$$


and

$$
\begin{aligned}
\Delta_{K k} & =\int_{E} d^{3} \mathbf{R} \mathbf{V}_{K}(\mathbf{R})^{*} \cdot \mathbf{A}_{k}(\mathbf{R}) \\
\Phi_{K k} & =\int_{E} d^{3} \mathbf{R} \mathbf{U}_{K}(\mathbf{R})^{*} \cdot \mathbf{A}_{k}(\mathbf{R}) .
\end{aligned}
$$

The integrals are over the external region. The matrices $G, H, \Delta, \Phi$ satisfy relations analogous to those for $C, D, \Gamma, \Lambda$.

The vector potential in general satifies the wave equation. Since the $Q_{n}$ or $R_{n}$ determine the vector potential inside the cavity, they also determine $\mathbf{A}$ and its derivatives on the boundary with the external region. Hence they also determine the field in the external region, which is specified by the $Q_{K}$ or $R_{K}$. However, the field in both regions is also specified by the $q_{k}$. Hence the sets $Q_{n}$ or $R_{n}, Q_{K}$ or $R_{K}$ are equivalent to the $q_{k}$ as generalised coordinates specifying the vector potential, and these coordinates are interconvertable. The inverse relationships are:

$$
q_{k}=\sum_{l, n} \mathbf{M}_{k l}^{-1} \Lambda_{n l}^{*} Q_{n}=\sum_{l, K} \mathbf{N}_{k l}^{-1} \Phi_{K l}^{*} Q_{K}=\sum_{l, n} \mathbf{M}_{k l}^{-1} \Gamma_{n l}^{*} R_{n}=\sum_{l, K} \mathbf{N}_{k l}^{-1} \Delta_{K l}^{*} R_{K}
$$

where the matrices $\mathbf{M}, \mathbf{N}$ are defined by cavity and external region integrals:

$$
\begin{aligned}
\mathbf{M}_{k l} & =\int_{C} d^{3} \mathbf{R} \mathbf{A}_{k}(\mathbf{R})^{*} \cdot \mathbf{A}_{l}(\mathbf{R}) \\
\mathbf{N}_{k l} & =\int_{E} d^{3} \mathbf{R} \mathbf{A}_{k}(\mathbf{R})^{*} \cdot \mathbf{A}_{l}(\mathbf{R}) .
\end{aligned}
$$

Clearly $\mathbf{M}_{k l}+\mathbf{N}_{k l}=\delta_{k l}$. The relationship in equations (31) illustrates the point that the number of true modes $k$ in the half space must equal the number of cavity NHM designated $n$ or the number of external NHM designated $K$. Hence the total number of true modes in the full space would be equal to twice the number of cavity or external NHM. Equivalently, the total number of true modes $\mathbf{A}_{k}$ in the full space would be equal to the number of cavity right travelling NHM $\mathbf{U}_{n}$ plus left travelling NHM $\mathbf{W}_{n}=\mathbf{V}_{n}^{*}$, or the number of right travelling $\mathbf{U}_{K}$ and left travelling $\mathbf{V}_{K}^{*}$ external NHM. This result is important in comparing the total number of quantum harmonic oscillators for the field described via true modes with that when it is described by NHM.

\subsection{Lagrangian}

The Lagrangian is defined by:

$$
L=\frac{1}{2} \epsilon_{0} \int d^{3} \mathbf{R}\left(\dot{\mathbf{A}}(\mathbf{R})^{2}-c^{2}[\boldsymbol{\nabla} \times \mathbf{A}(\mathbf{R})]^{2}\right) .
$$


The true mode expansion gives $L$ in terms of the generalised coordinates $q_{k}$ and velocities $\dot{q}_{k}$ (and their complex congugates) in the form:

$$
L=\epsilon_{0} \sum_{k}^{R}\left(\dot{q}_{k} \dot{q}_{k}^{*}-\omega_{k}^{2} q_{k} q_{k}^{*}\right) .
$$

The Lagrangian can also be written as:

$$
L=L_{C}+L_{E},
$$

the sum of contributions $L_{C}, L_{E}$ from the cavity and external regions. Using the NHM expansion the cavity contribution $L_{C}$ can be obtained in terms of either the generalised coordinates $Q_{n}$ and velocities $\dot{Q}_{n}$ or the alternative quantities $R_{n}$ and $\dot{R}_{n}$ (and their complex congugates). The external contribution $L_{E}$ involves $Q_{K}, \dot{Q}_{K}$ or $R_{K}, \dot{R}_{K}$. The expressions for these Lagrangians are:

$$
\begin{aligned}
& L_{C}=\epsilon_{0} \sum_{n}\left(\dot{Q}_{n} \dot{R}_{n}^{*}-\omega_{n}^{2} Q_{n} R_{n}^{*}\right)=\epsilon_{0} \sum_{n}\left(\dot{R}_{n} \dot{Q}_{n}^{*}-\omega_{n}^{2} R_{n} Q_{n}^{*}\right) \\
& L_{E}=\epsilon_{0} \sum_{K}\left(\dot{Q}_{K} \dot{R}_{K}^{*}-\omega_{K}^{2} Q_{K} R_{K}^{*}\right)=\epsilon_{0} \sum_{K}\left(\dot{R}_{K} \dot{Q}_{K}^{*}-\omega_{K}^{2} R_{K} Q_{K}^{*}\right) .
\end{aligned}
$$

For the cavity term the magnetic field integral has been evaluated approximately with derivatives of the slowly varying factors being neglected. The external NHM and HAM have been chosen so that the matrix $\Omega$ determining the magnetic energy contribution, which is given by:

$$
\begin{aligned}
\Omega_{K L} & =c^{2} \int_{E} d^{3} \mathbf{R}\left[\boldsymbol{\nabla} \times \mathbf{U}_{K}(\mathbf{R})\right]^{*} \cdot\left[\boldsymbol{\nabla} \times \mathbf{V}_{L}(\mathbf{R})\right] \\
& =\omega_{K}^{2} \delta_{K L}
\end{aligned}
$$

is in diagonal form with eigenvalues $\omega_{K}^{2}$. For the external NHM a wave number $k_{K}$ can be defined via $\omega_{K}=c k_{K}$.

\subsection{Generalised momenta}

The true mode generalised momenta are defined in terms of the Lagrangian and related to the generalised velocities as:

$$
\begin{aligned}
& p_{k}=\partial L / \partial \dot{q}_{k}^{*} \\
& p_{k}=\epsilon_{0} \dot{q}_{k} .
\end{aligned}
$$

In the left half space $p_{k *}=\left(p_{k}\right)^{*}$. We can also write $p_{k}$ as the sum of cavity and external terms as:

$$
\begin{aligned}
p_{k} & =p_{k}^{C}+p_{k}^{E} \\
p_{k}^{C} & =\partial L_{C} / \partial \dot{q}_{k}^{*} \quad p_{k}^{E}=\partial L_{E} / \partial \dot{q}_{k}^{*}
\end{aligned}
$$


For the cavity NHM it is convenient to introduce generalised momenta defined in terms of the cavity term in the Lagrangian via:

$$
P_{n}=\partial L_{C} / \partial\left(\dot{Q}_{n}\right)^{*} \quad S_{n}=\partial L_{C} / \partial\left(\dot{R}_{n}\right)^{*}
$$

rather than expressions involving derivatives of the total Lagrangian $L$. We are able to make this somewhat arbitary choice for $P_{n}$ and $S_{n}$ because our formal quantization process is based on the true mode quantities $p_{k}$ and $q_{k}$, and so only the $p_{k}$ need to be defined in the canonical way. The generalised momenta $P_{n}$ and $S_{n}$ are given by:

$$
\begin{aligned}
P_{n} & =\epsilon_{0} \sum_{m} \mathbf{C}_{n m} \dot{Q}_{m}=\epsilon_{0} \dot{R}_{n} \\
S_{n} & =\epsilon_{0} \sum_{m} \mathbf{D}_{n m} \dot{R}_{m}=\epsilon_{0} \dot{Q}_{n} .
\end{aligned}
$$

We note that the generalised momentum $P_{n}$ associated with the generalised coordinate $Q_{n}$ is given in terms of the generalised velocity $\dot{R}_{n}$ associated with the alternative coordinate $R_{n}$. The reverse applies as well, and these features are a direct consequence of the two mode functions $\mathbf{U}_{n}(\mathbf{R})$ and $\mathbf{V}_{n}(\mathbf{R})$ not

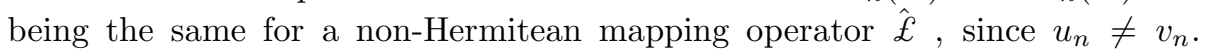
For the external region similar considerations give for the generalised momenta $P_{K}=\epsilon_{0} \dot{R}_{K}$ and $S_{K}=\epsilon_{0} \dot{Q}_{K}$. Together the sets of NHM generalised coordinates and momenta $Q_{n}, P_{n}$ or $R_{n}, S_{n}$ or $Q_{K}, P_{K}$ or $R_{K}, S_{K}$ are equivalent to the true mode generalised coordinates and momenta $q_{k}, p_{k}$. The cavity $P_{n}, S_{n}$ depend on the $p_{k}$. We have:

$$
P_{n}=\sum_{k}^{R} \Lambda_{n k} p_{k} \quad S_{n}=\sum_{k}^{R} \Gamma_{n k} p_{k}
$$

Interrelationships between the $P_{n}$ and the $S_{n}$ can easily be found in terms of C, D. These are:

$$
P_{n}=\sum_{m} \mathbf{C}_{n m} S_{m} \quad S_{n}=\sum_{m} \mathbf{D}_{n m} P_{m}
$$

Equations analogous to equations (48, 49) also apply for the $P_{K}, S_{K}$. The overall inverse relationships are:

$$
p_{k}=\sum_{l, n} \mathbf{M}_{k l}^{-1} \Gamma_{n l}^{*} P_{n}=\sum_{l, K} \mathbf{N}_{k l}^{-1} \Delta_{K l}^{*} P_{K}=\sum_{l, n} \mathbf{M}_{k l}^{-1} \Lambda_{n l}^{*} S_{n}=\sum_{l, K} \mathbf{N}_{k l}^{-1} \Phi_{K l}^{*} S_{K}
$$




\subsection{Conjugate momentum field}

The conjugate momentum field $\Pi(\mathbf{R})$ is defined as the derivative of the Lagrangian density with respect to $\dot{\mathbf{A}}$ and is given by:

$$
\Pi(\mathbf{R})=\varepsilon_{0} \dot{\mathbf{A}}(\mathbf{R}) .
$$

For all positions $\mathbf{R}$ it can be written as the sum of a right travelling light field $\Pi_{R}(\mathbf{R})$ and a left travelling field $\Pi_{R}(\mathbf{R})^{*}$. The generalised momenta acts as expansion coefficients for expressing the conjugate momentum field in terms of the mode functions. In terms of the true mode and NHM expansions involving the generalised momenta we find that:

$$
\begin{aligned}
\Pi(\mathbf{R}) & =\Pi_{R}(\mathbf{R})+\Pi_{R}(\mathbf{R})^{*} \\
\Pi_{R}(\mathbf{R}) & =\sum_{k}^{R} p_{k} \mathbf{A}_{k}(\mathbf{R}) \\
\Pi_{R}(\mathbf{R}) & =\sum_{n} S_{n} \mathbf{U}_{n}(\mathbf{R})=\sum_{n} P_{n} \mathbf{V}_{n}(\mathbf{R})
\end{aligned}
$$

with $\mathbf{R}$ inside the cavity in equations (54). Analogous expressions apply for the external region. Comparing the similar expression for the vector potential (equations (17)) we note the unexpected feature that the momentum $S_{n}$ is now the expansion coefficient for the NHM function $\mathbf{U}_{n}(\mathbf{R})$, whilst $P_{n}$ is that for the HAM function $\mathbf{V}_{n}(\mathbf{R})$. This role reversal for the generalised momenta is a consequence of introducing the bi-orthogonal NHM functions.

\subsection{Hamiltonian}

The Hamiltonian $H$ is defined via the true mode expression and obtained in terms of true mode generalised coordinates and momenta:

$$
\begin{aligned}
H & =\sum_{k}^{R}\left[p_{k} \dot{q}_{k}^{*}+p_{k}^{*} \dot{q}_{k}\right]-L \\
H & =\sum_{k}\left(p_{k} p_{k}^{*}+\epsilon_{0}^{2} \omega_{k}^{2} q_{k} q_{k}^{*}\right) / 2 \epsilon_{0} .
\end{aligned}
$$

The Hamiltonian is that for independent harmonic oscillators, one for each true mode in each half space. To obtain the Hamiltonian in terms of NHM we first write the Hamitonian as the sum of cavity and external contributions $H_{C}, H_{E}$

$$
H=H_{C}+H_{E}
$$

given by

$$
\begin{aligned}
& H_{C}=\sum_{k}^{R}\left[p_{k}^{C} \dot{q}_{k}^{*}+p_{k}^{C *} \dot{q}_{k}\right]-L_{C} \\
& H_{E}=\sum_{k}^{R}\left[p_{k}^{E} \dot{q}_{k}^{*}+p_{k}^{E *} \dot{q}_{k}\right]-L_{E}
\end{aligned}
$$


We then show that

$$
\begin{aligned}
& p_{k}^{C}=\sum_{n}\left(\Lambda_{n k}^{*} \dot{Q}_{n}+\Gamma_{n k}^{*} \dot{R}_{n}\right) / 2 \epsilon_{0} \\
& p_{k}^{E}=\sum_{K}\left(\Phi_{K k}^{*} \dot{Q}_{K}+\Delta_{K k}^{*} \dot{R}_{K}\right) / 2 \epsilon_{0}
\end{aligned}
$$

Equations (18) etc. then enable us to eliminate the $k$ sums involving $\dot{q}_{k}, \dot{q}_{k}^{*}$, so that $H_{C}, H_{E}$ now only contain NHM generalised velocities $\dot{Q}_{n}, \dot{R}_{n}, \dot{Q}_{K}, \dot{R}_{n}$. Eliminating all the NHM generalised velocities in favour of the NHM generalised momenta via $P_{n}=\epsilon_{0} \dot{R}_{n}, S_{n}=\epsilon_{0} \dot{Q}_{n}$ etc. finally gives the contributions to the Hamiltonian $H_{C}, H_{E}$ from the cavity and external regions. The cavity contribution $H_{C}$ only depends on $Q_{n}, P_{n}$ or $R_{n}, S_{n}$. and the external contribution $H_{E}$ only depends on $Q_{K}, P_{K}$ or $R_{K}, S_{K}$. Expressed in terms of just one set of generalised coordinates and momenta (the $Q, P$ or the $R, S$ choice), both $H_{C}$ and $H_{E}$ are Hamiltonians for coupled harmonic oscillators, two oscillators for each $n$ and $K$ and there will be double sums over $n$ and $K$. However if a mixture of both choices are used, the cavity and external region contributions to the Hamiltonian can be expressed as single sums:

$$
\begin{aligned}
& H_{C}=\sum_{n}\left(\left[P_{n}{ }^{*} S_{n}+S_{n}{ }^{*} P_{n}\right]+\epsilon_{0}^{2} \omega_{n}^{2}\left[Q_{n} R_{n}{ }^{*}+R_{n} Q_{n}{ }^{*}\right]\right) / 2 \epsilon_{0} \\
& H_{E}=\sum_{K}\left(\left[P_{K}{ }^{*} S_{K}+S_{K}{ }^{*} P_{K}\right]+\epsilon_{0}^{2} \omega_{K}^{2}\left[Q_{K} R_{K}{ }^{*}+R_{K} Q_{K}{ }^{*}\right]\right) / 2 \epsilon_{0} .
\end{aligned}
$$

Using the transformation relations in equations (21, 49), the sums over $n$ of the two terms in each of the square brackets in equation (62) can be shown to be equal, however we have chosen to leave the Hamiltonian in a more symmetrical form regarding the two sets of generalised position and momentum coordinates.

\subsection{Canonical quantization and commutation rules}

Canonical quantization involves replacing the generalised coordinates and momenta by quantum operators satisfying standard commutation rules. The replacements are:

$$
q_{k} \rightarrow \hat{q}_{k}, \quad q_{k}^{*} \rightarrow \hat{q}_{k}^{\dagger}=\hat{q}_{k *} \quad, p_{k} \rightarrow \hat{p}_{k}, \quad p_{k}^{*} \rightarrow \hat{p}_{k}^{\dagger}=\hat{p}_{k *}
$$

for true modes and

$$
\begin{aligned}
& Q_{n} \rightarrow \hat{Q}_{n}, \quad\left(Q_{n}\right)^{*} \rightarrow \hat{Q}_{n}^{\dagger} \quad, P_{n} \rightarrow \hat{P}_{n}, \quad\left(P_{n}\right)^{*} \rightarrow \hat{P}_{n}^{\dagger}, \\
& R_{n} \rightarrow \hat{R}_{n}, \quad\left(R_{n}\right)^{*} \rightarrow \hat{R}_{n}^{\dagger} \quad, S_{n} \rightarrow \hat{S}_{n}, \quad\left(S_{n}\right)^{*} \rightarrow \hat{S}_{n}^{\dagger}
\end{aligned}
$$

for the cavity NHM. The standard commutators that are non zero are:

$$
\left[\hat{q}_{k}, \hat{p}_{l}^{\dagger}\right]=\left[\hat{q}_{k}^{\dagger}, \hat{p}_{l}\right]=i \hbar \delta_{k l}
$$


from which the commutators for the cavity NHM operators can be obtained via the relationshps in equations $(18,48)$ and the properties for the $\Gamma, \Lambda$ matrices. For the cavity NHM operators the non zero results are:

$$
\left[\hat{Q}_{n}, \hat{P}_{m}^{\dagger}\right]=\left[\hat{Q}_{n}^{\dagger}, \hat{P}_{m}\right]=\left[\hat{R}_{n}, \hat{S}_{m}^{\dagger}\right]=\left[\hat{R}_{n}^{\dagger}, \hat{S}_{m}\right]=i \hbar \delta_{n m}
$$

and

$$
\begin{array}{ll}
{\left[\hat{Q}_{n}, \hat{S}_{m}^{\dagger}\right]=i \hbar \mathbf{D}_{n m}} & {\left[\hat{Q}_{n}^{\dagger}, \hat{S}_{m}\right]=i \hbar \mathbf{D}_{m n}} \\
{\left[\hat{R}_{n}, \hat{P}_{m}^{\dagger}\right]=i \hbar \mathbf{C}_{n m}} & {\left[\hat{R}_{n}^{\dagger}, \hat{P}_{m}\right]=i \hbar \mathbf{C}_{m n}}
\end{array}
$$

Similar replacements are made for the external NHM generalised coordinates and momenta, and commutation rules analogous to those for the $\hat{Q}_{n}, \hat{P}_{n}, \hat{R}_{n}, \hat{S}_{n}$ are obtained for the $\hat{Q}_{K}, \hat{P}_{K}, \hat{R}_{K}, \hat{S}_{K}$. The commutators involving both cavity and external NHM operators will be discussed below. The Hamiltonian $H$ becomes the operator $\hat{H}$ and the vector potential and conjugate momentum fields $\mathbf{A}(\mathbf{R}), \Pi(\mathbf{R})$ become field operators $\hat{\mathbf{A}}(\mathbf{R}), \hat{\Pi}(\mathbf{R})$. The formal expressions for these operators in terms of the generalised coordinate and momentum operators are exactly the same as in the classical expressions, so need not be repeated here.

The commutation rules for the field operators $\hat{\mathbf{A}}(\mathbf{R}), \hat{\Pi}(\mathbf{R})$ can be obtained via their true mode expressions and are given by:

$$
\left[\hat{\mathbf{A}}^{\alpha}(\mathbf{R}), \hat{\Pi}^{\beta}\left(\mathbf{R}^{\prime}\right)\right]=i \hbar \delta_{\alpha \beta} \delta\left(\mathbf{R}-\mathbf{R}^{\prime}\right)
$$

Those for the electric displacement field operator $\hat{\mathbf{D}}(\mathbf{R})=-\hat{\Pi}(\mathbf{R})$ and the magnetic field operator $\hat{\mathbf{B}}(\mathbf{R})=\nabla \times \hat{\mathbf{A}}(\mathbf{R})$ can then be obtained as:

$$
\left[\hat{\mathbf{B}}^{\alpha}(\mathbf{R}), \hat{\mathbf{D}}^{\beta}\left(\mathbf{R}^{\prime}\right)\right]=i \hbar \sum_{\gamma} \varepsilon_{\alpha \beta \gamma} \partial / \partial X_{\gamma} \delta\left(\mathbf{R}-\mathbf{R}^{\prime}\right)
$$

The commutators involving both cavity and external NHM operators are less straightforward to determine than those just involving one type at a time. One suitable approach involves using the commutation rules obtained for the electric displacement and magnetic field operators. The details of this calculation are of some interest, and are set out in the Appendix. The non zero results can be expressed in terms of surface integrals over the boundary $S$ between the cavity and external regions, and are given below as $I_{n K}, J_{n K}, K_{n K}, L_{n K}, \mathcal{I}_{n K}, \mathcal{J}_{n K}, \mathcal{K}_{n K}, \mathcal{L}_{n K}$. Apart from others obtained by taking the Hermitean adjoint, the non zero commutators are for the case of cavity generalised coordinates and external gener- 
alised momenta are:

$$
\begin{aligned}
& {\left[\hat{Q}_{n}, \hat{P}_{K}^{\dagger}\right]=\left(\hbar / k_{n}\right) \int_{S} d^{2} \mathbf{s} \mathbf{V}_{n}^{*} \cdot \mathbf{U}_{K}=i \hbar L_{n K}} \\
& {\left[\hat{R}_{n}, \hat{S}_{K}^{\dagger}\right]=\left(\hbar / k_{n}\right) \int_{S} d^{2} \mathbf{s} \mathbf{U}_{n}^{*} \cdot \mathbf{V}_{K}=i \hbar K_{n K}} \\
& {\left[\hat{Q}_{n}, \hat{S}_{K}^{\dagger}\right]=\left(\hbar / k_{n}\right) \int_{S} d^{2} \mathbf{s} \mathbf{V}_{n}^{*} \cdot \mathbf{V}_{K}=i \hbar J_{n K}} \\
& {\left[\hat{R}_{n}, \hat{P}_{K}^{\dagger}\right]=\left(\hbar / k_{n}\right) \int_{S} d^{2} \mathbf{s} \mathbf{U}_{n}^{*} \cdot \mathbf{U}_{K}=i \hbar I_{n K}}
\end{aligned}
$$

The non zero commutators for the case of cavity generalised momenta and external generalised coordinates are:

$$
\begin{aligned}
& {\left[\hat{P}_{n}, \hat{Q}_{K}^{\dagger}\right]=\left(i \hbar / k_{K}^{2}\right) \int_{S} d^{2} \mathbf{s} \hat{\mathbf{z}} \cdot \mathbf{U}_{n}^{*} \times\left(\boldsymbol{\nabla} \times \mathbf{V}_{K}\right)=-i \hbar \mathcal{K}_{n K}} \\
& {\left[\hat{S}_{n}, \hat{R}_{K}^{\dagger}\right]=\left(i \hbar / k_{K}^{2}\right) \int_{S} d^{2} \mathbf{s} \hat{\mathbf{z}} \cdot \mathbf{V}_{n}^{*} \times\left(\boldsymbol{\nabla} \times \mathbf{U}_{K}\right)=-i \hbar \mathcal{L}_{n K}} \\
& {\left[\hat{P}_{n}, \hat{R}_{K}^{\dagger}\right]=\left(i \hbar / k_{K}^{2}\right) \int_{S} d^{2} \mathbf{s} \hat{\mathbf{z}} \cdot \mathbf{U}_{n}^{*} \times\left(\boldsymbol{\nabla} \times \mathbf{U}_{K}\right)=-i \hbar \mathcal{I}_{n K}} \\
& {\left[\hat{S}_{n}, \hat{Q}_{K}^{\dagger}\right]=\left(i \hbar / k_{K}^{2}\right) \int_{S} d^{2} \mathbf{s} \hat{\mathbf{z}} \cdot \mathbf{V}_{n}^{*} \times\left(\boldsymbol{\nabla} \times \mathbf{V}_{K}\right)=-i \hbar \mathcal{J}_{n K}}
\end{aligned}
$$

These commutators are much smaller than those involving only cavity quantities or only external quantities.

\subsection{Annihilation and creation operators}

In view of the link between the various classical Hamiltonian terms and harmonic oscillators, it is appropriate to replace the generalised coordinate and momentum operators by annihilation and creation operators. For the true modes we define:

$$
\begin{aligned}
\hat{a}_{k}=\lambda_{k} \hat{q}_{k}+i \mu_{k} \hat{p}_{k} & \hat{a}_{k}^{\dagger}=\lambda_{k} \hat{q}_{k}^{\dagger}-i \mu_{k} \hat{p}_{k}^{\dagger} \\
\hat{a}_{k *}=\lambda_{k} \hat{q}_{k}^{\dagger}+i \mu_{k} \hat{p}_{k}^{\dagger} & \hat{a}_{k *}^{\dagger}=\lambda_{k} \hat{q}_{k}-i \mu_{k} \hat{p}_{k},
\end{aligned}
$$

where $\lambda_{k}=\sqrt{\epsilon_{0} \omega_{k} / 2 \hbar}$ and $\mu_{k}=\sqrt{1 / 2 \hbar \epsilon_{0} \omega_{k}}$. These have non zero commutation rules $\left[\hat{a}_{k}, \hat{a}_{l}^{\dagger}\right]=\delta_{k l}$ and the Hamiltonian operator can be written as the well known form

$$
\hat{H}=\sum_{k}\left(\hat{a}_{k}^{\dagger} \hat{a}_{k}+1 / 2\right) \cdot \hbar \omega_{k},
$$

corresponding to independent quantum harmonic oscillators, one for each true

mode in both half spaces. For the NHM a somewhat unusual choice is made 
owing to the juxtaposition of the $\hat{Q}, \hat{R}$ operators and $\hat{P}, \hat{S}$ operators in the Hamiltonian contributions $\hat{H}_{C}, \hat{H}_{E}$. We combine the $\hat{Q}$ with the $\hat{S}$ and the $\hat{R}$ with the $\hat{P}$ along with the Hermitean adjoints to define annihilation and creation operators.

For the cavity NHM it is possible to define eight operators of the annihilation and creation operator type:

$$
\begin{aligned}
\hat{A}_{n} & =\lambda_{n} \hat{Q}_{n}+i \mu_{n} \hat{S}_{n} & & \hat{A}_{n}^{\#}=\lambda_{n} \hat{R}_{n}^{\dagger}-i \mu_{n} \hat{P}_{n}^{\dagger} \\
\hat{B}_{n} & =\lambda_{n} \hat{R}_{n}^{\dagger}+i \mu_{n} \hat{P}_{n}^{\dagger} & & \hat{B}_{n}^{\#}=\lambda_{n} \hat{Q}_{n}-i \mu_{n} \hat{S}_{n} \\
\hat{A}_{n}^{\dagger} & =\lambda_{n} \hat{Q}_{n}^{\dagger}-i \mu_{n} \hat{S}_{n}^{\dagger} & & \hat{A}_{n}^{\# \dagger}=\lambda_{n} \hat{R}_{n}+i \mu_{n} \hat{P}_{n} \\
\hat{B}_{n}^{\dagger} & =\lambda_{n} \hat{R}_{n}-i \mu_{n} \hat{P}_{n} & & \hat{B}_{n}^{\# \dagger}=\lambda_{n} \hat{Q}_{n}^{\dagger}+i \mu_{n} \hat{S}_{n}^{\dagger},
\end{aligned}
$$

which are expressed as linear combinations of the eight original generalised position and momentum operators and their adjoints. With these choices we see that the annihilation, creation operator pairs are now $\left(\hat{A}_{n}, \hat{A}_{n}^{\#}\right),\left(\hat{B}_{n}, \hat{B}_{n}^{\#}\right),\left(\hat{A}_{n}^{\# \dagger}, \hat{A}_{n}^{\dagger}\right)$ and $\left(\hat{B}_{n}^{\# \dagger}, \hat{B}_{n}^{\dagger}\right)$ rather than the expected $\left(\hat{A}_{n}, \hat{A}_{n}^{\dagger}\right),\left(\hat{B}_{n}, \hat{B}_{n}^{\dagger}\right),\left(\hat{A}_{n}^{\# \dagger}, \hat{A}_{n}^{\#}\right)$ and $\left(\hat{B}_{n}^{\# \dagger}, \hat{B}_{n}^{\#}\right)$. The non zero commutation rules are:

$$
\left[\hat{A}_{n}, \hat{A}_{m}^{\#}\right]=\left[\hat{B}_{n}, \hat{B}_{m}^{\#}\right]=\left[\hat{A}_{n}^{\# \dagger}, \hat{A}_{m}^{\dagger}\right]=\left[\hat{B}_{n}^{\# \dagger}, \hat{B}_{m}^{\dagger}\right]=\delta_{n m}
$$

and

$$
\begin{array}{ll}
{\left[\hat{A}_{n}, \hat{A}_{m}^{\dagger}\right]=\mathbf{D}_{n m}} & {\left[\hat{B}_{n}^{\# \dagger}, \hat{B}_{m}^{\#}\right]=\mathbf{D}_{m n}} \\
{\left[\hat{B}_{n}, \hat{B}_{m}^{\dagger}\right]=\mathbf{C}_{m n}} & {\left[\hat{A}_{n}^{\# \dagger}, \hat{A}_{m}^{\#}\right]=\mathbf{C}_{n m}}
\end{array}
$$

Some commutators are zero because of the selection rule $\omega_{n}=\omega_{m}$ for non zero $\mathbf{C}_{n m}$ and $\mathbf{D}_{n m}$. Similar commutation rules occur in reference [19] (their $\hat{a}_{n}$ corresponds to our $\hat{A}_{n}$, their $\hat{b}_{n}^{\dagger}$ to our $\hat{A}_{n}^{\#}$ ), except that here we have twice as many annihilation, creation operator pairs since no operators corresponding to our $\hat{B}_{n}$ and $\hat{B}_{n}^{\#}$ occur. The precise nature of the eight operators can be seen by expressing each of them as a linear combination of the true mode annihilation and creation operators. Each turns out to actually involve both types of true mode operator, for example $\hat{A}_{n}$ is a linear combination of the $\hat{a}_{k}$ and the $\hat{a}_{k *}^{\dagger}$ with $k$ in the right half space. However, the expansion coefficient for one type of operator involves the factor $f_{-}\left(\omega_{n}, \omega_{k}\right)=\left(\omega_{n}-\omega_{k}\right) /\left(2 \sqrt{\omega_{n} \omega_{k}}\right)$, the other the factor $f_{+}\left(\omega_{n}, \omega_{k}\right)=\left(\omega_{n}+\omega_{k}\right) /\left(2 \sqrt{\omega_{n} \omega_{k}}\right)$. Assuming that all the field modes of interest have approximately the same frequency (monochromaticity approximation, cf. [19) terms involve the first factor can be ignored and the second factor approximated as unity. We then find the approximate relationships: 


$$
\begin{array}{rlrl}
\hat{A}_{n} & \cong \sum_{k}^{R} \Gamma_{n k} \hat{a}_{k} & \hat{A}_{n}^{\#} \cong \sum_{k}^{R} \Lambda_{n k}^{*} \hat{a}_{k}^{\dagger} \\
\hat{B}_{n} \cong \sum_{k}^{R} \Lambda_{n k}^{*} \hat{a}_{k *} & \hat{B}_{n}^{\#} \cong \sum_{k}^{R} \Gamma_{n k} \hat{a}_{k *}^{\dagger} \\
\hat{A}_{n}^{\# \dagger} \cong \sum_{k}^{R} \Lambda_{n k} \hat{a}_{k} & \hat{A}_{n}^{\dagger} \cong \sum_{k}^{R} \Gamma_{n k}^{*} \hat{a}_{k}^{\dagger} \\
\hat{B}_{n}^{\# \dagger} \cong \sum_{k}^{R} \Gamma_{n k}^{*} \hat{a}_{k *} & \hat{B}_{n}^{\dagger} \cong \sum_{k}^{R} \Lambda_{n k} \hat{a}_{k *}^{\dagger} .
\end{array}
$$

We thus can identify $\hat{A}_{n}$ and $\hat{B}_{n}$ as annihilation operators associated with right and left travelling modes respectively, and with $\hat{A}_{n}^{\#}$ and $\hat{B}_{n}^{\#}$ as their corresponding creation operators. A similar identification of $\hat{A}_{n}^{\# \dagger}$ and $\hat{B}_{n}^{\# \dagger}$ as annihilation operators, $\hat{A}_{n}^{\dagger}$ and $\hat{B}_{n}^{\dagger}$ the corresponding creation operators also applies. Using the selection rule $\omega_{n}=\omega_{m}$ for non zero $\mathbf{C}_{n m}$ and $\mathbf{D}_{n m}$, the eight operators for the cavity NHM can be interrelated as follows:

$$
\begin{aligned}
\hat{A}_{n}^{\# \dagger}=\sum_{m} \mathbf{C}_{n m} \hat{A}_{m} & \hat{B}_{n}^{\dagger}=\sum_{m} \mathbf{C}_{n m} \hat{B}_{m}^{\#} \\
\hat{A}_{n}^{\dagger}=\sum_{m} \mathbf{D}_{m n} \hat{A}_{m}^{\#} & \hat{B}_{n}^{\# \dagger}=\sum_{m} \mathbf{D}_{m n} \hat{B}_{m} .
\end{aligned}
$$

These relationships are used to simplify the cavity term in the Hamiltonian $\hat{H}_{C}$.

A similar approach can be used to construct annihilation and creation operators for the external NHM. For the external NHM we write:

$$
\begin{array}{rlrl}
\hat{A}_{K} & =\lambda_{K} \hat{Q}_{K}+i \mu_{K} \hat{S}_{K} & & \hat{A}_{K}^{\#}=\lambda_{K} \hat{R}_{K}^{\dagger}-i \mu_{K} \hat{P}_{K}^{\dagger} \\
\hat{B}_{K}=\lambda_{K} \hat{R}_{K}^{\dagger}+i \mu_{K} \hat{P}_{K}^{\dagger} & & \hat{B}_{K}^{\#}=\lambda_{K} \hat{Q}_{K}-i \mu_{K} \hat{S}_{K} \\
\hat{A}_{K}^{\dagger} & =\lambda_{K} \hat{Q}_{K}^{\dagger}-i \mu_{K} \hat{S}_{K}^{\dagger} & & \hat{A}_{K}^{\# \dagger}=\lambda_{K} \hat{R}_{K}+i \mu_{K} \hat{P}_{K} \\
\hat{B}_{K}^{\dagger} & =\lambda_{K} \hat{R}_{K}-i \mu_{K} \hat{P}_{K} & & \hat{B}_{K}^{\# \dagger}=\lambda_{K} \hat{Q}_{K}^{\dagger}+i \mu_{K} \hat{S}_{K}^{\dagger} .
\end{array}
$$

The commutation rules are analogous to those for the cavity NHM operators, except that some further commutators are non zero because the selection rule $\omega_{K}=\omega_{L}$ for non zero $\mathbf{G}_{K L}$ and $\mathbf{H}_{K L}$ is only approximate, and the expected results like $\left[\hat{A}_{K}, \hat{A}_{L}^{\dagger}\right]=\mathbf{H}_{K L}$ are multiplied by the factor $f_{+}\left(\omega_{K}, \omega_{L}\right)$ that is only approximately unity. Approximate relations between the external NHM annihilation and creation operators and the true mode operators that are analogous to equations (86) and interrelations between the external NHM operators that are analogous to equations (87) can be obtained. These depend respectively, 
on the monochromaticity assumption and the approximate selection rules on $\mathbf{G}_{K L}, \mathbf{H}_{K L}$. Operators such as $\hat{A}_{K}$ and $\hat{B}_{K}$ are approximate linear combinations of true mode annihilation operators, with $\hat{A}_{K}^{\#}$ and $\hat{B}_{K}^{\#}$ their corresponding creation operators.

\section{$2.9 \quad$ Field operators}

The field operators $\hat{\mathbf{A}}(\mathbf{R}), \hat{\Pi}(\mathbf{R})$ can be now expressed in terms of annihilation and creation operators. In terms of true modes we have:

$$
\begin{aligned}
\hat{\mathbf{A}}(\mathbf{R}) & =\sum_{k}^{R} \sqrt{\hbar / 2 \epsilon_{0} \omega_{k}}\left(\left[\hat{a}_{k}+\hat{a}_{k *}^{\dagger}\right] \mathbf{A}_{k}(\mathbf{R})+\left[\hat{a}_{k}^{\dagger}+\hat{a}_{k *}\right] \mathbf{A}_{k}(\mathbf{R})^{*}\right) \\
& =\sum_{k} \sqrt{\hbar / 2 \epsilon_{0} \omega_{k}}\left(\hat{a}_{k} \mathbf{A}_{k}(\mathbf{R})+\hat{a}_{k}^{\dagger} \mathbf{A}_{k}(\mathbf{R})^{*}\right)
\end{aligned}
$$

and

$$
\begin{aligned}
\hat{\Pi}(\mathbf{R}) & =\sum_{k}^{R} \frac{1}{i} \sqrt{\hbar \epsilon_{0} \omega_{k} / 2}\left(\left[\hat{a}_{k}-\hat{a}_{k *}^{\dagger}\right] \mathbf{A}_{k}(\mathbf{R})-\left[\hat{a}_{k}^{\dagger}-\hat{a}_{k *}\right] \mathbf{A}_{k}(\mathbf{R})^{*}\right) \\
& =\sum_{k} \frac{1}{i} \sqrt{\hbar \epsilon_{0} \omega_{k} / 2}\left(\hat{a}_{k} \mathbf{A}_{k}(\mathbf{R})-\hat{a}_{k}^{\dagger} \mathbf{A}_{k}(\mathbf{R})^{*}\right)
\end{aligned}
$$

for all positions R. Expanding in NHM functions the field operators are:

$$
\begin{aligned}
& \hat{\mathbf{A}}(\mathbf{R})=\sum_{n} \sqrt{\hbar / 2 \epsilon_{0} \omega_{n}}\left(\left[\hat{A}_{n}+\hat{B}_{n}^{\#}\right] \mathbf{U}_{n}(\mathbf{R})+\left[\hat{A}_{n}^{\#}+\hat{B}_{n}\right] \mathbf{V}_{n}(\mathbf{R})^{*}\right) \\
& \hat{\Pi}(\mathbf{R})=\sum_{n} \frac{1}{i} \sqrt{\hbar \epsilon_{0} \omega_{n} / 2}\left(\left[\hat{A}_{n}-\hat{B}_{n}^{\#}\right] \mathbf{U}_{n}(\mathbf{R})-\left[\hat{A}_{n}^{\#}-\hat{B}_{n}\right] \mathbf{V}_{n}(\mathbf{R})^{*}\right)
\end{aligned}
$$

for positions $\mathbf{R}$ in the cavity region. The monochromaticity approximation is not required. Comparing equations $(89$, 91) with equations $(93$, 94) again suggests the identification of $\hat{A}_{n}$ and $\hat{B}_{n}$ as annihilation operators associated with right and left travelling modes respectively, and also indicates that we have twice as many annihilation operators as in reference [19]. Their expressions corresponding to equations $(93,94)$ do not include the $\hat{B}_{n}^{\#}$ and $\hat{B}_{n}$ contributions.

\subsection{Quantum Hamiltonian}

The cavity term in the Hamiltonian can be expressed in terms of the cavity NHM annihilation and creation operators. We find that: 


$$
\begin{aligned}
\hat{H}_{C} & =\sum_{n} \hbar \omega_{n}\left\{\left(\frac{1}{2}\left[\hat{A}_{n}^{\#} \hat{A}_{n}+\hat{A}_{n}^{\dagger} \hat{A}_{n}^{\# \dagger}\right]+\frac{1}{2}\right)+\left(\frac{1}{2}\left[\hat{B}_{n}^{\#} \hat{B}_{n}+\hat{B}_{n}^{\dagger} \hat{B}_{n}^{\# \dagger}\right]+\frac{1}{2}\right)\right\} \\
& =\sum_{n} \hbar \omega_{n}\left\{\left(\hat{A}_{n}^{\dagger} \hat{A}_{n}^{\# \dagger}+\frac{1}{2}\right)+\left(\hat{B}_{n}^{\dagger} \hat{B}_{n}^{\# \dagger}+\frac{1}{2}\right)\right\} \\
& =\sum_{n} \hbar \omega_{n}\left\{\left(\hat{A}_{n}^{\#} \hat{A}_{n}+\frac{1}{2}\right)+\left(\hat{B}_{n}^{\#} \hat{B}_{n}+\frac{1}{2}\right)\right\}
\end{aligned}
$$

The first form is obtained just by inverting equations (82) and substituting into the operator form of equation (62). The derivation of the second and third forms for $\hat{H}_{C}$ uses equations (87), which is based on the selection rule $\omega_{n}=\omega_{m}$ for non zero $\mathbf{C}_{n m}$ and $\mathbf{D}_{n m}$. The final expression for the cavity contribution $\hat{H}_{C}$ to the Hamiltonian turns out to be the Hamiltonian for a set of independent quantum harmonic oscillators (QHO), two for each cavity NHM index $n$ - one corresponding to the left travelling NHM, the other to the right travelling NHM.. The lack of coupling between the cavity NHM QHO follows from the result that all $\hat{A}_{n}^{\#} \hat{A}_{n}$ and $\hat{B}_{n}^{\#} \hat{B}_{n}$ commute with each other. Because of the commutation rules, the quantities $\hat{A}_{n}^{\#} \hat{A}_{n}$ and $\hat{B}_{n}^{\#} \hat{B}_{n}$ act as number operators, even though they are not Hermitean. However, although the individual terms in equation (96) are not Hermitean, the overall sum giving $\hat{H}_{C}$ is. The existence of QHO will become apparent when the approximate energy eigenstates for the Hamiltonian $\hat{H}$ are obtained.

Expressions for the field operators in the external region analogous to equations (93, 94) apply however without using the approximate relations between the external NHM annihilation and creation operators and the true mode operators that are analogous to equations (86) and the interrelationships between the external NHM operators that are analogous to equations (87), and more importantly the external region contribution to the Hamiltonian $\hat{H}_{E}$ can be written as:

$$
\hat{H}_{E}=\sum_{K} \hbar \omega_{K}\left\{\left(\frac{1}{2}\left[\hat{A}_{K}^{\#} \hat{A}_{K}+\hat{A}_{K}^{\dagger} \hat{A}_{K}^{\# \dagger}\right]+\frac{1}{2}\right)+\left(\frac{1}{2}\left[\hat{B}_{K}^{\#} \hat{B}_{K}+\hat{B}_{K}^{\dagger} \hat{B}_{K}^{\# \dagger}\right]+\frac{1}{2}\right)\right\}
$$

If we now apply the relationships that analogous to equations (87), the last expression can be simplified to give an approximate form for $\hat{H}_{E}$ :

$$
\hat{H}_{E}^{0}=\sum_{K} \hbar \omega_{K}\left\{\left(\hat{A}_{K}^{\#} \hat{A}_{K}+\frac{1}{2}\right)+\left(\hat{B}_{K}^{\#} \hat{B}_{K}+\frac{1}{2}\right)\right\}
$$

The approximate external region contribution $\hat{H}_{E}^{0}$ to the field Hamiltonian will be seen to be the Hamiltonian for a set of independent QHOs, two for each external region NHM $K$. Independence follows from all the $\hat{A}_{K}^{\#} \hat{A}_{K}$ and $\hat{B}_{K}^{\#} \hat{B}_{K}$ 
factors commuting with each other. Again, the non Hermitean quantities $\hat{A}_{K}^{\#} \hat{A}_{K}$ and $\hat{B}_{K}^{\#} \hat{B}_{K}$ act as number operators. The full expression for the external region contribution to the Hamiltonian can then be written as the sum of the harmonic oscillator term $\hat{H}_{E}^{0}$ and a coupling term $\hat{V}_{E}$ as follows:

$$
\hat{H}_{E}=\hat{H}_{E}^{0}+\hat{V}_{E}
$$

where the coupling term $\hat{V}_{E}$ is

$$
\hat{V}_{E}=\frac{1}{2} \sum_{K} \hbar \omega_{K}\left\{\hat{A}_{K}^{\dagger} \hat{A}_{K}^{\# \dagger}+\hat{B}_{K}^{\dagger} \hat{B}_{K}^{\# \dagger}\right\}-\frac{1}{2} \sum_{K} \hbar \omega_{K}\left\{\hat{A}_{K}^{\#} \hat{A}_{K}+\hat{B}_{K}^{\#} \hat{B}_{K}\right\}
$$

It should be noted that neither $\hat{H}_{E}^{0}$ nor $\hat{V}_{E}$ are Hermitean, though their sum giving $\hat{H}_{E}$ is. It is also possible to write:

$$
\hat{H}_{E}=\left(\hat{H}_{E}^{0}\right)^{\dagger}+\left(\hat{V}_{E}\right)^{\dagger}
$$

where:

$$
\begin{aligned}
\left(\hat{H}_{E}^{0}\right)^{\dagger}= & \sum_{K} \hbar \omega_{K}\left\{\left(\hat{A}_{K}^{\dagger} \hat{A}_{K}^{\# \dagger}+\frac{1}{2}\right)+\left(\hat{B}_{K}^{\dagger} \hat{B}_{K}^{\# \dagger}+\frac{1}{2}\right)\right\} \\
\left(\hat{V}_{E}\right)^{\dagger}= & \frac{1}{2} \sum_{K} \hbar \omega_{K}\left\{\hat{A}_{K}^{\#} \hat{A}_{K}+\hat{B}_{K}^{\#} \hat{B}_{K}\right\} \\
& -\frac{1}{2} \sum_{K} \hbar \omega_{K}\left\{\hat{A}_{K}^{\dagger} \hat{A}_{K}^{\# \dagger}+\hat{B}_{K}^{\dagger} \hat{B}_{K}^{\# \dagger}\right\}
\end{aligned}
$$

The field Hamiltonian $\hat{H}$ is now given by:

$$
\hat{H}=\hat{H}_{C}+\hat{H}_{E}=\hat{H}_{C}+\hat{H}_{E}^{0}+\hat{V}_{E}=\hat{H}_{C}+\left(\hat{H}_{E}^{0}\right)^{\dagger}+\left(\hat{V}_{E}\right)^{\dagger} .
$$

Although there are similarities in that the quantum field Hamiltonian is the sum of non-commuting cavity and external region contributions, the final form of our Hamiltonian for the EM field differs from that in reference [21]. Here there are no off-diagonal terms so the Hamiltonian is simpler.

\subsection{Non-commutation of cavity and external NHM op- erators}

The commutation rules for either the cavity NHM or the external region NHM annihilation and creation operators separately have been described. Since the field Hamiltonian is the sum of cavity and external region contributions, each of which depend on their own annihilation and creation operators, it is also important to consider the commutators between cavity and external region operators. These can be obtained from those for the generalised coordinates and momenta and hence depend on certain surface integrals, as we have seen in 
equations $(72-79)$. The commutators are listed in tables 1 and 2 . In these tables $a_{n K}=(1 / 2) \sqrt{\omega_{n} / \omega_{K}}$ and $b_{n K}=(1 / 2) \sqrt{\omega_{K} / \omega_{n}}$. Another thirty-two commutation rules can be obtained by taking the adjoints of the results in tables 1 and 2.

\begin{tabular}{||l||l||l|l||l||}
\hline \hline$[\hat{L}, \hat{R}]$ & $\hat{A}_{K}$ & $\hat{A}_{K}^{\#}$ & $\hat{B}_{K}$ & $\hat{B}_{K}^{\#}$ \\
\hline \hline$\hat{A}_{n}$ & 0 & $\begin{array}{l}a_{n K} L_{n K} \\
+b_{n K} \mathcal{L}_{n K}\end{array}$ & $\begin{array}{l}-a_{n K} L_{n K} \\
+b_{n K} \mathcal{L}_{n K}\end{array}$ & 0 \\
\hline$\hat{A}_{n}^{\#}$ & $\begin{array}{l}-a_{n K} K_{n K}^{*} \\
-b_{n K} \mathcal{K}_{n K}^{*}\end{array}$ & 0 & 0 & $\begin{array}{l}a_{n K} K_{n K}^{*} \\
-b_{n K} \mathcal{K}_{n K}^{*}\end{array}$ \\
\hline$\hat{B}_{n}$ & $\begin{array}{l}-a_{n K} K_{n K}^{*} \\
+b_{n K} \mathcal{K}_{n K}^{*}\end{array}$ & 0 & 0 & $\begin{array}{l}a_{n K} K_{n K}^{*} \\
+b_{n K} \mathcal{K}_{n K}^{*}\end{array}$ \\
\hline$\hat{B}_{n}^{\#}$ & 0 & $\begin{array}{l}a_{n K} L_{n K} \\
-b_{n K} \mathcal{L}_{n K}\end{array}$ & $\begin{array}{l}-a_{n K} L_{n K} \\
-b_{n K} \mathcal{L}_{n K}\end{array}$ & 0 \\
\hline
\end{tabular}

Table 1. Commutators for cavity and external NHM annihilation and creation operators.

\begin{tabular}{||l||l||l||l||l||}
\hline \hline$[\hat{L}, \hat{R}]$ & $\hat{A}_{K}^{\dagger}$ & $\hat{A}_{K}^{\# \dagger}$ & $\hat{B}_{K}^{\dagger}$ & $\hat{B}_{K}^{\# \dagger}$ \\
\hline \hline$\hat{A}_{n}$ & $\begin{array}{l}a_{n K} J_{n K} \\
+b_{n K} \mathcal{J}_{n K}\end{array}$ & 0 & 0 & $\begin{array}{l}-a_{n K} J_{n K} \\
+b_{n K} \mathcal{J}_{n K}\end{array}$ \\
\hline$\hat{A}_{n}^{\#}$ & 0 & $\begin{array}{l}-a_{n K} I_{n K}^{*} \\
-b_{n K} \mathcal{I}_{n K}^{*}\end{array}$ & $\begin{array}{l}a_{n K} I_{n K}^{*} \\
-b_{n K} \mathcal{I}_{n K}^{*}\end{array}$ & 0 \\
\hline$\hat{B}_{n}$ & 0 & $\begin{array}{l}-a_{n K} I_{n K}^{*} \\
+b_{n K} \mathcal{I}_{n K}^{*}\end{array}$ & $\begin{array}{l}a_{n K} I_{n K}^{*} \\
+b_{n K} \mathcal{I}_{n K}^{*}\end{array}$ & 0 \\
\hline$\hat{B}_{n}^{\#}$ & $\begin{array}{l}a_{n K} J_{n K} \\
-b_{n K} \mathcal{J}_{n K}\end{array}$ & 0 & 0 & $-a_{n K} J_{n K}$ \\
& & & $-b_{n K} \mathcal{J}_{n K}$ \\
\hline
\end{tabular}

Table 2. Commutators for cavity and adjoints of external NHM annihilation and creation operators.

\subsection{Energy eigenstates for unperturbed Hamiltonians}

Several possible choices are available for a suitable unperturbed Hamiltonian for which approximate energy eigenstates can be obtained. One choice is the cavity region Hamiltonian $\hat{H}_{C}$, another is the external region approximate Hamiltonian $\hat{H}_{E}^{0}$. A third choice is the external region approximate Hamiltonian $\left(\hat{H}_{E}^{0}\right)^{\dagger}$. In the case of $\hat{H}_{C}$ the non-Hermitean quantities $\hat{A}_{n}^{\#} \hat{A}_{n}, \hat{B}_{n}^{\#} \hat{B}_{n}$ behave as a set of commuting number operators for which we can find simultaneous left and right eigenstates, even though the individual number operators are non-Hermitean. These eigenstates are also eigenstates of $\hat{H}_{C}$. Similarly, in the case of $\hat{H}_{E}^{0}$ the non-Hermitean quantities $\hat{A}_{K}^{\#} \hat{A}_{K}, \hat{B}_{K}^{\#} \hat{B}_{K}$ again behave as a set of commuting number operators for which we can find simultaneous left and right eigenstates, even though the individual number operators are non-Hermitean. These eigenstates are also eigenstates of $\hat{H}_{E}^{0}$. In each case, the unperturbed Hamiltonian involves the annihilation, creation operator pairs $\left(\hat{A}_{n}, \hat{A}_{n}^{\#}\right),\left(\hat{B}_{n}, \hat{B}_{n}^{\#}\right)$ or $\left(\hat{A}_{K}, \hat{A}_{K}^{\#}\right),\left(\hat{B}_{K}, \hat{B}_{K}^{\#}\right)$ and the true mode vacuum state $|0>=| \ldots 0_{k} \ldots . .0_{k *} . .>$ acts approximately as a vacuum state for the NHM 
annihilation operators $\hat{A}_{n}, \hat{B}_{n}$ or $\hat{A}_{K}, \hat{B}_{K}$, with

$$
\hat{A}_{n}\left|0>=\hat{B}_{n}\right| 0>=\hat{A}_{K}\left|0>=\hat{B}_{K}\right| 0>=0
$$

(see equations (86)). The right simultaneous eigenstates of the number operators $\hat{A}_{n}^{\#} \hat{A}_{n}, \hat{B}_{n}^{\#} \hat{B}_{n}$ or $\hat{A}_{K}^{\#} \hat{A}_{K}, \hat{B}_{K}^{\#} \hat{B}_{K}$ can be constructed in the standard form as:

$$
\begin{gathered}
\left|\left\{n_{n}\right\},\left\{m_{n}\right\}>=\prod_{n} \frac{\left(\hat{A}_{n}^{\#}\right)^{n_{n}}}{\sqrt{n_{n} !}} \frac{\left(\hat{B}_{n}^{\#}\right)^{m_{n}}}{\sqrt{m_{n} !}}\right| \mathbf{0}>, \\
\left|\left\{n_{K}\right\},\left\{m_{K}\right\}>=\prod_{K} \frac{\left(\hat{A}_{K}^{\#}\right)^{n_{K}}}{\sqrt{n_{K} !}} \frac{\left(\hat{B}_{K}^{\#}\right)^{m_{K}}}{\sqrt{m_{K} !}}\right| \mathbf{0}>
\end{gathered}
$$

where $n_{n}, m_{n}, n_{K}, m_{K}$ are all positive integers. These states are also right eigenstates for the unperturbed Hamiltonians $\hat{H}_{C}$ or $\hat{H}_{E}^{0}$, where (with $\left\{\mathbf{N}_{C}\right\} \equiv$ $\left\{n_{n}\right\},\left\{m_{n}\right\}$ and $\left.\left\{\mathbf{N}_{E}\right\}=\left\{n_{K}\right\},\left\{m_{K}\right\}\right)$

$$
\begin{aligned}
& \hat{H}_{C}\left|\left\{\mathbf{N}_{C}\right\}>=E\left(\left\{\mathbf{N}_{C}\right\}\right)\right|\left\{\mathbf{N}_{C}\right\}>, \\
& \hat{H}_{E}^{0}\left|\left\{\mathbf{N}_{E}\right\}>=E\left(\left\{\mathbf{N}_{E}\right\}\right)\right|\left\{\mathbf{N}_{E}\right\}>
\end{aligned}
$$

and the energy is given by:

$$
\begin{aligned}
E\left(\left\{n_{n}\right\},\left\{m_{n}\right\}\right) & =\sum_{n} \hbar \omega_{n}\left(n_{n}+m_{n}+1\right) \\
E\left(\left\{n_{K}\right\},\left\{m_{K}\right\}\right) & =\sum_{K} \hbar \omega_{K}\left(n_{K}+m_{K}+1\right)
\end{aligned}
$$

These are clearly quantum harmonic oscillator energy expressions. For the state $\mid\left\{n_{n}\right\},\left\{m_{n}\right\}>$ there are $n_{n}, m_{n}$ photons associated with the cavity NHMs $U_{n}(R), V_{n}(R)^{*}$, and for the state $\mid\left\{n_{K}\right\},\left\{m_{K}\right\}>$ there are $n_{K}, m_{K}$ photons associated with the external region NHMs $U_{K}(R), V_{K}(R)^{*}$. As the number operators $\hat{A}_{n}^{\#} \hat{A}_{n}, \hat{B}_{n}^{\#} \hat{B}_{n}$ or $\hat{A}_{K}^{\#} \hat{A}_{K}, \hat{B}_{K}^{\#} \hat{B}_{K}$ are non-Hermitean, the left simultaneous eigenstates of these operators are different to the right eigenstates. However, both the left and right eigenstates are also eigenstates of the unperturbed Hamiltonian $\hat{H}_{C}$ or $\hat{H}_{E}^{0}$, having the same energy as in equations (110, 111). The left eigenstates are obtained in the form:

$$
\begin{aligned}
\left(\left\{n_{n}\right\},\left\{m_{n}\right\} \mid\right. & =<\mathbf{0} \mid \prod_{n} \frac{\left(\hat{A}_{n}\right)^{n_{n}}}{\sqrt{n_{n} !}} \frac{\left(\hat{B}_{n}\right)^{m_{n}}}{\sqrt{m_{n} !}}, \\
\left(\left\{n_{K}\right\},\left\{m_{K}\right\} \mid\right. & =<\mathbf{0} \mid \prod_{K} \frac{\left(\hat{A}_{K}\right)^{n_{K}}}{\sqrt{n_{K} !}} \frac{\left(\hat{B}_{K}\right)^{m_{K}}}{\sqrt{m_{K} !}}
\end{aligned}
$$


and the eigenstates for $\hat{H}_{C}$ satisfy orthonormality and completeness relations:

$$
\begin{aligned}
\left(\left\{\mathbf{N}_{C}\right\} \mid\left\{\mathbf{N}_{C}^{/}\right\}\right. & >\boldsymbol{\delta}\left(\left\{\mathbf{N}_{C}\right\},\left\{\mathbf{N}_{C}^{/}\right\}\right) \\
\sum_{\left\{\mathbf{N}_{C}\right\}} \mid\left\{\mathbf{N}_{C}\right\} & >\left(\left\{\mathbf{N}_{C}\right\} \mid=1\right.
\end{aligned}
$$

in an obvious notation. The round bracket notation for the left eigenstates has been introduced to distinguish $\left(\left\{\mathbf{N}_{C}\right\} \mid\right.$ from the adjoint of the right eigenstates, $\left(\mid\left\{\mathbf{N}_{C}\right\}>\right)^{\dagger}=<\left\{\mathbf{N}_{C}\right\} \mid$, whose role will be discussed below. Analogous results apply for the states based on the external Hamiltonian $\hat{H}_{E}^{0}$. Similar results have been obtained in reference [19].

Some simple states will be specified as examples of these expressions. The zero photon or vacuum state is the same as the true mode vacuum. The one photon states are:

$$
\begin{array}{ll}
\left|1_{n}^{A}>=\hat{A}_{n}^{\#}\right| \mathbf{0}> & \left|1_{n}^{B}>=\hat{B}_{n}^{\#}\right| \mathbf{0}> \\
\left|1_{K}^{A}>=\hat{A}_{K}^{\#}\right| \mathbf{0}> & \left|1_{K}^{B}>=\hat{B}_{K}^{\#}\right| \mathbf{0}>.
\end{array}
$$

For the cavity Hamiltonian $\hat{H}_{C}$ there are two other choices of right and left eigenstates, which are the adjoints of the states $\left(\left\{n_{n}\right\},\left\{m_{n}\right\} \mid\right.$ and $\mid\left\{n_{n}\right\},\left\{m_{n}\right\}>$ and are given by:

$$
\begin{aligned}
\left.\mid\left\{n_{n}\right\},\left\{m_{n}\right\}\right) & =\prod_{n} \frac{\left(\hat{A}_{n}^{\dagger}\right)^{n_{n}}}{\sqrt{n_{n} !}} \frac{\left(\hat{B}_{n}^{\dagger}\right)^{m_{n}}}{\sqrt{m_{n} !}} \mid \mathbf{0}> \\
& <\left\{n_{n}\right\},\left\{m_{n}\right\}|=<\mathbf{0}| \prod_{n} \frac{\left(\hat{A}_{n}^{\# \dagger}\right)^{n_{n}}}{\sqrt{n_{n} !}} \frac{\left(\hat{B}_{n}^{\# \dagger}\right)^{m_{n}}}{\sqrt{m_{n} !}} .
\end{aligned}
$$

Both states have energies given by equation (110) and the new right eigenvalue equation is:

$$
\left.\left.\hat{H}_{C} \mid\left\{\mathbf{N}_{C}\right\}\right)=E\left(\left\{\mathbf{N}_{C}\right\}\right) \mid\left\{\mathbf{N}_{C}\right\}\right) .
$$

The new orthogonality and completeness relationships that apply are:

$$
\begin{aligned}
& \left.<\left\{\mathbf{N}_{C}\right\} \mid\left\{\mathbf{N}_{C}^{/}\right\}\right)=\boldsymbol{\delta}\left(\left\{\mathbf{N}_{C}\right\},\left\{\mathbf{N}_{C}^{/}\right\}\right) \\
\left.\sum_{\left\{\mathbf{N}_{C}\right\}} \mid\left\{\mathbf{N}_{C}\right\}\right) & <\left\{\mathbf{N}_{C}\right\} \mid=1 .
\end{aligned}
$$

These results may be obtained directly by taking the adjoints of previous equations or by using the alternative form for $\hat{H}_{C}$ in equation (95), noting that $\hat{A}_{n}^{\dagger} \hat{A}_{n}^{\# \dagger}$ and $\hat{B}_{n}^{\dagger} \hat{B}_{n}^{\# \dagger}$ are also number operators and that $\hat{A}_{n}^{\# \dagger}\left|0>=\hat{B}_{n}^{\# \dagger}\right| 0>=0$ using equation (86).

For the approximate external region Hamiltonian the situation is similar. Here the additional right and left eigenstates of the Hermitean adjoint $\left(\hat{H}_{E}^{0}\right)^{\dagger}$ 
are $\left.\mid\left\{n_{K}\right\},\left\{m_{K}\right\}\right)$ and $<\left\{n_{K}\right\},\left\{m_{K}\right\} \mid$, which are the adjoints of $\left(\left\{n_{K}\right\},\left\{m_{K}\right\} \mid\right.$ and $\mid\left\{n_{K}\right\},\left\{m_{K}\right\}>$ respectively and can be obtained from equations (113, 107). Both states have energies given by equation (111) but the right eigenvalue equation now involves $\left(\hat{H}_{E}^{0}\right)^{\dagger}$ and is:

$$
\left.\left.\left(\hat{H}_{E}^{0}\right)^{\dagger} \mid\left\{\mathbf{N}_{E}\right\}\right)=E\left(\left\{\mathbf{N}_{E}\right\}\right) \mid\left\{\mathbf{N}_{E}\right\}\right) .
$$

Orthogonality and completeness relationships analogous to the cavity case apply, and the results are obtained similarly.

\section{Atom-field interactions and spontaneous de- cay}

\subsection{Rotating wave approximation and coupling constants}

In the electric dipole approximation the interaction between an atom and the field is given by $\hat{V}=\hat{\mathbf{d}} \cdot \hat{\Pi} / \epsilon_{0}$, where $\hat{\mathbf{d}}$ is the atomic dipole operator and the conjugate momentum field $\hat{\Pi}$ is evaluated at the atomic position (see reference (四). The dipole operator can be written as the sum of a component $\hat{\mathbf{d}}^{+}$associated with upward atomic transitions and a component $\hat{\mathbf{d}}^{-}$associated with downward atomic transitions, where $\hat{\mathbf{d}}^{+}=\left(\hat{\mathbf{d}}^{-}\right)^{\dagger}$. The conjugate momentum field can be expressed as the sum of its positive and negative frequency components, $\hat{\Pi}^{+}, \hat{\Pi}^{-}$respectively; Thus we have:

$$
\begin{aligned}
\hat{\mathbf{d}} & =\hat{\mathbf{d}}^{+}+\hat{\mathbf{d}}^{-} \\
\hat{\Pi} & =\hat{\Pi}^{+}+\hat{\Pi}^{-} .
\end{aligned}
$$

Expressions for the positive and negative frequency component for positions $\mathbf{R}$ in the cavity are:

$$
\begin{aligned}
& \hat{\Pi}^{+}=\sum_{n} \frac{1}{i} \sqrt{\hbar \epsilon_{0} \omega_{n} / 2}\left(\hat{A}_{n} \mathbf{U}_{n}(\mathbf{R})+\hat{B}_{n} \mathbf{V}_{n}(\mathbf{R})^{*}\right) \\
& \hat{\Pi}^{-}=-\sum_{n} \frac{1}{i} \sqrt{\hbar \epsilon_{0} \omega_{n} / 2}\left(\hat{B}_{n}^{\#} \mathbf{U}_{n}(\mathbf{R})+\hat{A}_{n}^{\#} \mathbf{V}_{n}(\mathbf{R})^{*}\right)=\left(\hat{\Pi}^{+}\right)^{\dagger}
\end{aligned}
$$

Note that although $\hat{\Pi}^{-}=\left(\hat{\Pi}^{+}\right)^{\dagger}$ overall, the same is not true on a term by term basis. In the rotating wave approximation (RWA) the atom-field interaction is given by:

$$
\hat{V}_{R W A}=\left(\hat{\mathbf{d}}^{+} \cdot \hat{\Pi}^{+}+\hat{\mathbf{d}}^{-} \cdot \hat{\Pi}^{-}\right) / \boldsymbol{\epsilon}_{0} .
$$

For a two level atom (TLA) with upper state $|e\rangle$, lower state $|g\rangle$, the dipole operator components are: $\hat{\mathbf{d}}^{ \pm}=\mathbf{d} \hat{\sigma}_{ \pm}$, where $\mathbf{d}$ is the dipole matrix element $<e|\hat{\mathbf{d}}| g>$ and $\hat{\sigma}_{+}=|e><g|, \hat{\sigma}_{-}=|g><e|$ are the upward, downward 
transition operators. The atomic transition energy is $\hbar \omega_{0}$. For the TLA located in the cavity at position $\mathbf{R}$, the RWA atom-field interaction can be expressed as:

$$
\hat{V}_{R W A}=\hat{\sigma}_{+}\left[\sum_{n} \hbar\left(g_{n}^{A} \hat{A}_{n}+g_{n}^{B} \hat{B}_{n}\right)\right]-\hat{\sigma}_{-}\left[\sum_{n} \hbar\left(g_{n}^{A} \hat{B}_{n}^{\#}+g_{n}^{B} \hat{A}_{n}^{\#}\right)\right]
$$

where the coupling constants are defined by:

$$
\begin{aligned}
& g_{n}^{A}=-i \sqrt{\omega_{n} / 2 \hbar \epsilon_{0}}\left(\mathbf{d} \cdot \mathbf{U}_{n}(\mathbf{R})\right) \\
& g_{n}^{B}=-i \sqrt{\omega_{n} / 2 \hbar \epsilon_{0}}\left(\mathbf{d} \cdot \mathbf{V}_{n}(\mathbf{R})^{*}\right) .
\end{aligned}
$$

These coupling constants are also referred to as one-photon Rabi frequencies. Here we note one of the unusual features of the NHM theory - whereas an upward atomic transition accompanied by the absorption of a $\mathbf{U}_{n}(\mathbf{R})$ photon will be associated with the coupling constant $g_{n}^{A}$, a downward atomic transition accompanied by the emission of a $\mathbf{U}_{n}(\mathbf{R})$ photon will be associated with the opposite coupling constant $g_{n}^{B}$. The situation involving the absorption or emission of a $\mathbf{V}_{n}(\mathbf{R})^{*}$ photon is similar, with the opposite coupling constant $g_{n}^{A}$ again being associated with emission. Thus absorption and emission involve different coupling constants, a peculiarity ultimately responsible for the Petermann factor.

\subsection{Decay of excited atom}

The spontaneous emission problem for the TLA located in the cavity will be treated via a simple approach using the essential states approximation and the RWA. In addition, the effect of the external region term $\hat{H}_{E}$ will be neglected in the first instance, where we just focus on the initial emission of photons into the cavity NHM. The external region term $\hat{H}_{E}$ comes into play when the loss of cavity NHM photons and the creation of external NHM photons is considered that is, cavity loss processes. As this implies, we are assuming that the cavity loss rate is slow compared to the spontaneous emission rate from the atom into the cavity. Thus we will take as our Hamiltonian:

$$
\hat{H}_{T}=\hat{H}_{A}+\hat{H}_{C}+\hat{V}_{R W A},
$$

where $\hat{H}_{A}$ is the atomic Hamiltonian and ignoring the zero point energy terms as usual. Hence our essential states will just include product states of the form: $|e>| \mathbf{0}>,|g>| 1_{n}^{A}>$ and $|g>| 1_{n}^{B}>$. The initial state is given by:

$$
|\Psi(0)>=| e>\mid \mathbf{0}>
$$

and the time dependent Schrodinger state vector is written as: 


$$
\begin{aligned}
\mid \Psi(t) & >=C_{e}(t) \exp \left(-i \omega_{0} t / 2\right)|e>| \mathbf{0}> \\
+\sum_{n} C_{n}^{A}(t) \exp \left(-i\left[\omega_{n}-\omega_{0} / 2\right] t\right) \mid g & >\mid 1_{n}^{A}> \\
+\sum_{n} C_{n}^{B}(t) \exp \left(-i\left[\omega_{n}-\omega_{0} / 2\right] t\right) \mid g & >\mid 1_{n}^{B}>
\end{aligned}
$$

where $C_{e}(t), C_{n}^{A}(t)$ and $C_{n}^{B}(t)$ are amplitudes in the interaction picture. Using the time dependent Schrodinger equation we obtain coupled equations for the amplitudes:

$$
\begin{aligned}
i \frac{d C_{e}(t)}{d t} & =-\sum_{n}\left\{g_{n}^{A} \exp \left(i \delta_{n} t\right) C_{n}^{A}(t)+g_{n}^{B} \exp \left(i \delta_{n} t\right) C_{n}^{B}(t)\right\} \\
i \frac{d C_{n}^{A}(t)}{d t} & =g_{n}^{B} \exp \left(-i \delta_{n} t\right) C_{e}(t) \\
i \frac{d C_{n}^{B}(t)}{d t} & =g_{n}^{A} \exp \left(-i \delta_{n} t\right) C_{e}(t),
\end{aligned}
$$

where the detuning is $\delta_{n}=\omega_{0}-\omega_{n}$. Note the opposite coupling constants in the last two equations. The one photon amplitudes $C_{n}^{A}(t), C_{n}^{B}(t)$ can be formally eliminated to give a single integro-differential equation for the excited state amplitude $C_{e}(t)$ of the form:

$$
\frac{d C_{e}(t)}{d t}=\int_{0}^{t} d \tau K(\tau) C_{e}(t-\tau)
$$

where the kernel $K(\tau)$ is defined by:

$$
K(\tau)=\sum_{n} 2 g_{n}^{A} g_{n}^{B} \exp \left(i \delta_{n} \tau\right)
$$

In view of the convolution integral form, the equation for $C_{e}(t)$ can be solved in the general case via Laplace transform methods in terms of the Laplace transform of the kernel:

$$
\tilde{K}(s)=i \sum_{n} 2 g_{n}^{A} g_{n}^{B} /\left(\delta_{n}+i s\right) .
$$

Recalling that our generic index $n$ specfies the angular frequency $\omega_{n}$, the polarization $\boldsymbol{\alpha}_{n}$ and the transverse mode index $\theta_{n}$, we may convert the sum over $n$ in equation (139) to an integral over $\omega_{n}$ times a mode density $\rho\left(\omega_{n}\right)$, together with sums over $\boldsymbol{\alpha}_{n}$ and $\theta_{n}$. Then, assuming that the coupling constants $g_{n}^{A}, g_{n}^{B}$ are slowly varying with frequency $\omega_{n}$, we see that the kernel $K(\tau)$ decreases to zero over a correlation time $\tau_{c}$ of order the inverse bandwidth of the coupling constants. Further, assuming this time scale is short compared to the decay time 
$T_{e}$ of the excited state amplitude, we can then make the Markoff approximation. In this case:

$$
\frac{d C_{e}(t)}{d t} \simeq\left\{\int_{0}^{\infty} d \tau K(\tau)\right\} C_{e}(t)
$$

showing that the probability of the atom being excited, $P_{e}(t)=\left|C_{e}(t)\right|^{2}$ decays exponentially to zero with a rate $\Gamma_{e}$ given by:

$$
\Gamma_{e}=-2 \operatorname{Re} \tilde{K}(\epsilon)
$$

where $\epsilon$ is small.

To evaluate $\Gamma_{e}$, equation (14) can be used to interrelate the coupling constants in equation (140), giving

$$
g_{n}^{B}=-\sum_{m} \mathbf{D}_{n m}\left(g_{m}^{A}\right)^{*}
$$

which is then substituted into equation (140). Now the transformation matrix $\mathbf{D}_{n m}$ is zero unless the frequency and polarizations are the same $\left(\omega_{n}=\omega_{m}, \boldsymbol{\alpha}_{n}=\right.$ $\left.\boldsymbol{\alpha}_{m}\right)$, so after replacing the sums over $n$ and $m$ by an integral over $\omega_{n}$ involving NHM density $\rho\left(\omega_{n}\right)$ and by sums over $\boldsymbol{\alpha}_{n}$ and the transverse mode indices $\theta_{n}, \theta_{m}$, we then obtain for $\tilde{K}(\epsilon)$ the expression:

$$
\begin{aligned}
\tilde{K}(\epsilon)= & i \int d \omega_{n} \rho\left(\omega_{n}\right) 2 \sum_{\boldsymbol{\alpha}_{n}} \sum_{\theta_{n}} \sum_{\theta_{m}}\left(g_{n}^{A}\right) \mathbf{D}_{n m}\left(g_{m}^{A}\right)^{*} \frac{P}{\left(\omega_{n}-\omega_{0}\right)} \\
& -\pi \int d \omega_{n} \rho\left(\omega_{n}\right) 2 \sum_{\alpha_{n}} \sum_{\theta_{n}} \sum_{\theta_{m}}\left(g_{n}^{A}\right) \mathbf{D}_{n m}\left(g_{m}^{A}\right)^{*} \delta\left(\omega_{n}-\omega_{0}\right)(1
\end{aligned}
$$

From the Hermiteancy properties of $\mathbf{D}_{n m}$ it is not difficult to see that the double sum $\sum_{\theta_{n}} \sum_{\theta_{m}}\left(g_{n}^{A}\right) \mathbf{D}_{n m}\left(g_{m}^{A}\right)^{*}$ is real, so that in general we have for the decay rate:

$$
\begin{aligned}
\Gamma_{e}= & 4 \pi \rho\left(\omega_{0}\right) \sum_{\boldsymbol{\alpha}_{n}} \sum_{\theta_{n}}\left|g_{n}^{A}\left(\omega_{0}\right)\right|^{2} K_{n}\left(\omega_{0}\right) \\
& +4 \pi \rho\left(\omega_{0}\right) \sum_{\boldsymbol{\alpha}_{n}} \sum_{\theta_{n} \neq \theta_{m}} \sum_{\theta_{m} \neq \theta_{n}} g_{n}^{A}\left(\omega_{0}\right) \mathbf{D}_{n m}\left(\omega_{0}\right) g_{m}^{A}\left(\omega_{0}\right)^{*},
\end{aligned}
$$

where we have substituted the Petermann factors $K_{n}\left(\omega_{0}\right)$ for the diagonal matrix elements $\mathbf{D}_{n n}\left(\omega_{0}\right)$. Note that all (diagonal and off-diagonal) transformation matrix elements $\mathbf{D}_{n m}$ with $\boldsymbol{\alpha}_{n}=\boldsymbol{\alpha}_{m}$ are independent of $\boldsymbol{\alpha}_{n}$. In general then, the decay rate $\Gamma_{e}$ will involve the cavity NHM coupling constants $g_{n}^{A}$ and the transformation matrix elements $\mathbf{D}_{n m}$ evaluated at the atomic transition frequency $\omega_{0}$. The decay rate is the sum of diagonal terms involving the Petermann factors $K_{n}\left(\omega_{0}\right)=\mathbf{D}_{n n}\left(\omega_{0}\right)$ and off-diagonal terms involving the general 
$\mathbf{D}_{n m}\left(\omega_{0}\right)$ with $\theta_{n} \neq \theta_{m}$. However, if the transformation matrix is such that the sums over $\theta_{n}, \theta_{m}$ are dominated by the contribution coming from a single (real) diagonal element $\mathbf{D}_{n n}$ with transverse mode index $\theta_{n}$, then the other terms can be ignored, leading to the following simple result for the decay rate:

$$
\begin{aligned}
\Gamma_{e} & =4 \pi \rho\left(\omega_{0}\right) \sum_{\alpha_{n}}\left|g_{n}^{A}\left(\omega_{0}, \theta_{n}\right)\right|^{2} \mathbf{D}_{n n}\left(\omega_{0}, \theta_{n}\right) \\
& =K_{n} \Gamma_{e}^{F}
\end{aligned}
$$

where

$$
\Gamma_{e}^{F}=4 \pi \rho\left(\omega_{0}\right) \sum_{\alpha_{n}}\left|g_{n}^{A}\left(\omega_{0}, \theta_{n}\right)\right|^{2}
$$

is the decay rate expected for a normal cavity situation. Thus we see for this special case where a single NHM dominates, the decay rate has been enhanced by the Petermann factor $K_{n}$.

\section{Conclusion}

The present paper is a fully quantum treatment of the field for unstable optical systems. A standard canonical quantization proceedure is used, based on expanding the vector potential in the unstable cavity region and in the external region via non-Hermitean (Fox-Li) modes and their Hermitean adjoint modes, all defined via the optical system. Three dimensional systems are treated, using the paraxial 22] and monochromaticity approximations [19] for the cavity non-Hermitean modes. Both right and left travelling modes are included. The results are similar to [19], 21] but differ in detail. The field is equivalent to a set of quantum harmonic oscillators (QHO), associated now with non-Hermitean modes rather than true modes, and thus confirming the validity of the photon model for the case of unstable optical systems. The annihilation, creation operator pairs for each QHO are not Hermitean adjoints. A doubling of the number of annihilation, creation operator pairs occurs compared to [19], 21], showing that the total number of true mode QHO's equals the total number of QHO's associated either with the cavity or the external region non-Hermitean modes. The final form of our quantum Hamiltonian for the EM field is the sum of noncommuting cavity and external field contributions. To a good approximation, both the cavity and external field Hamiltonians can be expressed as a sum of independent QHO Hamiltonians for each non-Hermitean mode, but the external field Hamiltonian also includes a coupling term responsible for external nonHermitean mode photon exchange processes. The two independent QHO Hamiltonians are alternative choices for an unperturbed Hamiltonian. Left and right eigenstates for each of these unperturbed Hamiltonians are obtained, and the energy is given by the usual QHO result. Certain cavity non-Hermitean mode annihilation, creation operators do not commute with others for the external 
region non-Hermitean modes, leading to cavity energy gain and loss processes. A simple description of the cavity-external region light coupling in terms of surface integrals involving products of cavity and external non-Hermitean mode functions is found.

Atom-field interactions are treated in the electric dipole and rotating wave approximations. Atomic transitions leading to cavity non-Hermitean mode photon absorption are associated with a different coupling constant to that for atomic transitions leading to photon emission. This feature is directly related to the treatment being based on non-Hermitean mode functions and leads to enhanced emission rates. Using the essential states approach, the spontaneous decay of a two level atom located in the cavity is treated. The external region term in the field Hamiltonian is neglected assuming that atomic decay into the cavity is much faster than cavity decay. Coupled equations for the amplitudes of atom-field states involving the excited atom and no photons or the ground state atom with one photon in a cavity non-Hermitean mode are obtained. Markovian decay occurs under certain conditions, the decay rate being enhanced by the Petermann factor [12] in special cases when a single NHM dominates.

\section{Acknowledgements}

This work was supported by the Engineering and Physical Sciences Research Council (U.K.). Helpful discussions with Professor M. Babiker, Professor S. M. Barnett, Professor P. D. Drummond, Dr. S. Dutra, Dr. B. M. Garraway, Professor P. L. Knight, Dr. C. Lamprecht, Dr. P. W. Milonni, Professor G. C. H. New, Dr. G. Nienhuis, Professor H. Ritsch and Professor S. Stenholm are gratefully acknowledged.

\section{Appendix A: Commutation rules for cavity and external region operators}

\subsection{Commutation rules for $\hat{D}$ and $\hat{B}$ field operators}

To derive the commutation rules between cavity NHM generalised coordinates or momenta and external region NHM generalised momenta or coordinates we use the general rules for the magnetic field and electric displacement operators:

$$
\begin{aligned}
{\left[\hat{\mathbf{B}}^{\alpha}(\mathbf{R}), \hat{\mathbf{D}}^{\beta}\left(\mathbf{R}^{\prime}\right)\right] } & =i \hbar \varepsilon_{\alpha \beta \gamma} \partial / \partial X_{\gamma} \delta\left(\mathbf{R}-\mathbf{R}^{\prime}\right) \\
{\left[\hat{\mathbf{D}}^{\alpha}(\mathbf{R}), \hat{\mathbf{B}}^{\beta}\left(\mathbf{R}^{\prime}\right)\right] } & =-i \hbar \varepsilon_{\alpha \beta \gamma} \partial / \partial X_{\gamma} \delta\left(\mathbf{R}-\mathbf{R}^{\prime}\right),
\end{aligned}
$$


region we will use the lowest order terms in the small parameter $f$ for both 
$\hat{\mathbf{B}}(\mathbf{R})$ and $\hat{\mathbf{D}}(\mathbf{R})$ namely:

$$
\begin{aligned}
\hat{\mathbf{B}}(\mathbf{R}) & =\sum_{n}\left(i k_{n} \hat{Q}_{n} \hat{\mathbf{z}} \times \mathbf{U}_{n}(\mathbf{R})-i k_{n} \hat{R}_{n}^{\dagger} \hat{\mathbf{z}} \times \mathbf{V}_{n}^{*}(\mathbf{R})\right) \\
\hat{\mathbf{D}}(\mathbf{R}) & =-\sum_{n}\left(\hat{S}_{n} \mathbf{U}_{n}(\mathbf{R})+\hat{P}_{n}^{\dagger} \mathbf{V}_{n}^{*}(\mathbf{R})\right),
\end{aligned}
$$

whilst in the external region we will write:

$$
\begin{aligned}
\hat{\mathbf{D}}\left(\mathbf{R}^{\prime}\right) & =-\sum_{K}\left(\hat{S}_{K} \mathbf{U}_{K}\left(\mathbf{R}^{\prime}\right)+\hat{P}_{K}^{\dagger} \mathbf{V}_{K}^{*}\left(\mathbf{R}^{\prime}\right)\right) \\
\hat{\mathbf{B}}\left(\mathbf{R}^{\prime}\right) & =\sum_{K}\left(\hat{Q}_{K} \nabla \times \mathbf{U}_{K}\left(\mathbf{R}^{\prime}\right)+\hat{R}_{K}^{\dagger} \nabla \times \mathbf{V}_{K}^{*}\left(\mathbf{R}^{\prime}\right)\right) .
\end{aligned}
$$

For simplicity the cavity will be taken to be the region between the planes $z=z_{0}$ and $z=z_{b}+\epsilon$ and the external region to be the region between $z=z_{b}-\epsilon$ and $z=\infty$. The quantity $\epsilon$ is a small distance to allow for the cavity and external regions to overlap slightly, and will be taken to zero afterwards. The plane $z=z_{b}$ is the boundary between the cavity and external regions, which are shown in figure 2 .

\subsection{Cases of cavity NHM coordinates and external NHM momenta}

The commutation rules between cavity NHM coordinates and external region momenta are found by substituting for $\hat{\mathbf{B}}(\mathbf{R})$ and $\hat{\mathbf{D}}\left(\mathbf{R}^{\prime}\right)$ from equations (150, 152) into equation (148), which gives:

$$
\begin{aligned}
i \hbar \sum_{\gamma} \varepsilon_{\alpha \beta \gamma} \partial / \partial X_{\gamma} \delta\left(\mathbf{R}-\mathbf{R}^{\prime}\right)=-\sum_{\gamma m(\gamma)} & \sum_{L}\left\{i k_{m} \varepsilon_{\alpha z \gamma} U_{m}(\mathbf{R}) U_{L}^{\beta}\left(\mathbf{R}^{\prime}\right)\left[\hat{Q}_{m}, \hat{S}_{L}\right]\right. \\
& +i k_{m} \varepsilon_{\alpha z \gamma} U_{m}(\mathbf{R}) V_{L}^{\beta *}\left(\mathbf{R}^{\prime}\right)\left[\hat{Q}_{m}, \hat{P}_{L}^{\dagger}\right] \\
& -i k_{m} \varepsilon_{\alpha z \gamma} V_{m}^{*}(\mathbf{R}) U_{L}^{\beta}\left(\mathbf{R}^{\prime}\right)\left[\hat{R}_{m}^{\dagger}, \hat{S}_{L}\right] \\
& \left.-i k_{m} \varepsilon_{\alpha z \gamma} V_{m}^{*}(\mathbf{R}) V_{L}^{\beta *}\left(\mathbf{R}^{\prime}\right)\left[\hat{R}_{m}^{\dagger}, \hat{P}_{L}^{\dagger}\right]\right\}
\end{aligned}
$$

On the right side the sum over $\gamma$ only involves $x, y$ and that over $m$ only those with $\hat{\boldsymbol{\alpha}}_{m}=\hat{\boldsymbol{\gamma}}$. For such terms the components of $\mathbf{U}_{m}$ and $\mathbf{V}_{m}^{*}$ are $U_{m}$ and $V_{m}^{*}$.

\subsubsection{Case of $\left[\hat{Q}_{n}, \hat{P}_{K}^{\dagger}\right]$}

To obtain the commutation rule for $\left[\hat{Q}_{n}, \hat{P}_{K}^{\dagger}\right]$ we multiply each side of equation (154) by $V_{n}^{*}(\mathbf{R}) U_{K}^{\beta}\left(\mathbf{R}^{\prime}\right)$, integrate $\mathbf{R}, \mathbf{R}^{\prime}$ over the cavity and external regions respectively, then sum over $\beta$. Using the biorthogonality results in equations 
(10, 26) and neglecting integrals where the complex conjugation occurs either zero or two times we find that:

$i \hbar \sum_{\beta \gamma} \varepsilon_{\alpha \beta \gamma} \int_{C} d^{3} \mathbf{R} \int_{E} d^{3} \mathbf{R}^{/} V_{n}^{*}(\mathbf{R}) U_{K}^{\beta}\left(\mathbf{R}^{\prime}\right) \partial / \partial X_{\gamma} \delta\left(\mathbf{R}-\mathbf{R}^{\prime}\right)=-\sum_{\gamma=\alpha_{n}} i k_{n} \varepsilon_{\alpha z \gamma}\left[\hat{Q}_{n}, \hat{P}_{K}^{\dagger}\right]$.

There are only two non-zero possibilities $\hat{\boldsymbol{\alpha}}=\hat{\mathbf{x}}$ or $\hat{\boldsymbol{\alpha}}=\hat{\mathbf{y}}$. These give the two equations:

$$
\begin{aligned}
i k_{n}\left[\hat{Q}_{n}, \hat{P}_{K}^{\dagger}\right] .= & i \hbar \int_{C} d^{3} \mathbf{R} \int_{E} d^{3} \mathbf{R}^{\prime} V_{n}^{*}(\mathbf{R}) U_{K}^{y}\left(\mathbf{R}^{\prime}\right) \partial / \partial z \delta\left(\mathbf{R}-\mathbf{R}^{\prime}\right) \\
& -i \hbar \int_{C} d^{3} \mathbf{R} \int_{E} d^{3} \mathbf{R}^{/} V_{n}^{*}(\mathbf{R}) U_{K}^{z}\left(\mathbf{R}^{\prime}\right) \partial / \partial y \delta\left(\mathbf{R}-\mathbf{R}^{\prime}\right)
\end{aligned}
$$

with $\hat{\boldsymbol{\alpha}}_{n}=\hat{\mathbf{y}}$ and

$$
\begin{aligned}
-i k_{n}\left[\hat{Q}_{n}, \hat{P}_{K}^{\dagger}\right] .= & i \hbar \int_{C} d^{3} \mathbf{R} \int_{E} d^{3} \mathbf{R}^{\prime} V_{n}^{*}(\mathbf{R}) U_{K}^{z}\left(\mathbf{R}^{\prime}\right) \partial / \partial x \delta\left(\mathbf{R}-\mathbf{R}^{\prime}\right) \\
& -i \hbar \int_{C} d^{3} \mathbf{R} \int_{E} d^{3} \mathbf{R}^{/} V_{n}^{*}(\mathbf{R}) U_{K}^{x}\left(\mathbf{R}^{\prime}\right) \partial / \partial z \delta\left(\mathbf{R}-\mathbf{R}^{\prime}\right)
\end{aligned}
$$

with $\hat{\boldsymbol{\alpha}}_{n}=\hat{\mathbf{x}}$.

Considering the double integral on the right side of equation (157) involving $\partial / \partial x$, this is of the form:

$$
\begin{aligned}
I_{x}= & \int_{C} d y d z \int_{E} d^{3} \mathbf{R}^{\prime} \delta\left(y-y^{\prime}\right) \delta\left(z-z^{\prime}\right) U_{K}^{z}\left(\mathbf{R}^{\prime}\right) \\
& \cdot \int_{C} d x V_{n}^{*}(\mathbf{R}) \partial / \partial x \delta\left(x-x^{\prime}\right) \\
= & \int_{C} d y d z \int_{E} d^{3} \mathbf{R}^{\prime} \delta\left(y-y^{\prime}\right) \delta\left(z-z^{\prime}\right) U_{K}^{z}\left(\mathbf{R}^{\prime}\right) \\
& \quad \int_{C} d x\left\{\partial / \partial x\left[\delta\left(x-x^{\prime}\right) V_{n}^{*}(\mathbf{R})\right]-\delta\left(x-x^{\prime}\right) \partial / \partial x\left[V_{n}^{*}(\mathbf{R})\right]\right\},
\end{aligned}
$$

using partial integration. For the first term carrying out the $x$ integration leads to a result dependent on $V_{n}^{*}(\mathbf{R})$ for $x=-\infty$ and $x=+\infty$. As the NHM functions eventually go to zero for large $x$, this contribution can be ignored. For the remaining term we now have:

$$
\begin{aligned}
& I_{x}=-\int_{C} d^{3} \mathbf{R} \int_{E} d^{3} \mathbf{R}^{\prime} \delta\left(\mathbf{R}-\mathbf{R}^{\prime}\right) U_{K}^{z}\left(\mathbf{R}^{\prime}\right) \partial / \partial x\left[V_{n}^{*}(\mathbf{R})\right] \\
&=-\int_{C} d^{2} \mathbf{s} \int_{E} d^{2} \mathbf{s} / \delta\left(\mathbf{s}-\mathbf{s}^{\prime}\right) U_{K}^{z}\left(\mathbf{s}^{\prime}, z_{b}\right) \partial / \partial x\left[V_{n}^{*}\left(\mathbf{s}, z_{b}\right)\right] \\
& \cdot \int_{z_{0}}^{z_{b}+\epsilon} d z \int_{z_{b}-\epsilon}^{\infty} d z^{\prime} \delta\left(z-z^{\prime}\right),
\end{aligned}
$$


where in view of the $\delta\left(\mathbf{R}-\mathbf{R}^{/}\right)$factor, the ordinary functions can be expressed in terms of their values on the boundary, where $z=z_{b}$. The integrals over $z$ and $z^{\prime}$ give a result proportional to the small quantity $\epsilon$, and thus the integral $I_{x}$ is zero. Similar considerations show that the term involving $\partial / \partial y$ on the right side of equation (156) is also zero. The two equations $(156,157)$ are now both the same as:

$$
\left[\hat{Q}_{n}, \hat{P}_{K}^{\dagger}\right] .=\left(\hbar / k_{n}\right) \int_{C} d^{3} \mathbf{R} \int_{E} d^{3} \mathbf{R}^{/} \mathbf{V}_{n}^{*}(\mathbf{R}) \cdot \mathbf{U}_{K}\left(\mathbf{R}^{\prime}\right) \partial / \partial z \delta\left(\mathbf{R}-\mathbf{R}^{\prime}\right)
$$

since for the case where $\hat{\boldsymbol{\alpha}}_{n}=\hat{\mathbf{x}}$ the scalar product picks out the component $U_{K}^{x}\left(\mathbf{R}^{\prime}\right)$ and for the case $\hat{\boldsymbol{\alpha}}_{n}=\hat{\mathbf{y}}$ the scalar product picks out the component $U_{K}^{y}\left(\mathbf{R}^{/}\right)$.

Considering the double integral on the right side of equation 160 involving $\partial / \partial z$, this is of the form:

$$
\begin{aligned}
I_{z}= & -\int_{C} d^{3} \mathbf{R} \int_{E} d^{3} \mathbf{R}^{/} \mathbf{V}_{n}^{*}(\mathbf{R}) \cdot \mathbf{U}_{K}\left(\mathbf{R}^{\prime}\right) \partial / \partial z\left[\delta\left(\mathbf{R}-\mathbf{R}^{\prime}\right)\right] \\
= & \int_{C} d x d y \int_{E} d^{3} \mathbf{R}^{\prime} \delta\left(x-x^{\prime}\right) \delta\left(y-y^{\prime}\right) \mathbf{U}_{K}\left(\mathbf{R}^{\prime}\right) \\
& \cdot \int_{C} d z\left\{\partial / \partial z\left[\delta\left(z-z^{\prime}\right) \mathbf{V}_{n}^{*}(\mathbf{R})\right]-\delta\left(z-z^{\prime}\right) \partial / \partial z\left[\mathbf{V}_{n}^{*}(\mathbf{R})\right]\right\} \\
= & \int_{C} d^{2} \mathbf{s} \int_{E} d^{2} \mathbf{s}^{/} \delta\left(\mathbf{s}-\mathbf{s}^{\prime}\right) \int_{z_{b}-\epsilon}^{\infty} d z^{/} \mathbf{U}_{K}\left(\mathbf{s}^{\prime}, z^{\prime}\right) \\
& \cdot \int_{z_{0}}^{z_{b}+\epsilon} d z\left\{\partial / \partial z\left[\delta\left(z-z^{\prime}\right) \mathbf{V}_{n}^{*}(\mathbf{s}, z)\right]-\delta\left(z-z^{\prime}\right) \partial / \partial z\left[\mathbf{V}_{n}^{*}(\mathbf{s}, z)\right]\right\}
\end{aligned}
$$

using partial integration and then specifying the limits of integration over $z$ and $z^{\prime}$. The integration over $z$ in the first term is carried out and unlike the similar integrations over $x$ or $y$ that applied to the $I_{x}$ or $I_{y}$ cases we find that a non-zero contribution results from the $z=z_{b}+\epsilon$ limit. The second term is similar to the 
other term in the $I_{x}$ or $I_{y}$ cases and overall we now have:

$$
\begin{aligned}
& I_{z}= \int_{C} d^{2} \mathbf{s} \int_{E} d^{2} \mathbf{s} / \delta\left(\mathbf{s}-\mathbf{s}^{\prime}\right) \int_{z_{b}-\epsilon}^{\infty} d z^{\prime} \mathbf{U}_{K}\left(\mathbf{s}^{\prime}, z^{\prime}\right) \\
& \cdot\left\{\delta\left(z_{b}+\epsilon-z^{\prime}\right) \mathbf{V}_{n}^{*}\left(\mathbf{s}, z_{b}+\epsilon\right)\right\} \\
&-\int_{C} d^{2} \mathbf{s} \int_{E} d^{2} \mathbf{s}^{\prime} \delta\left(\mathbf{s}-\mathbf{s}^{\prime}\right) \int_{z_{b}-\epsilon}^{\infty} d z^{\prime} \mathbf{U}_{K}\left(\mathbf{s}^{\prime}, z^{\prime}\right) \\
& \cdot \int_{z_{0}}^{z_{b}+\epsilon} d z\left\{\delta\left(z-z^{\prime}\right) \partial / \partial z\left[\mathbf{V}_{n}^{*}(\mathbf{s}, z)\right]\right\} \\
&= \int_{C} d^{2} \mathbf{s} \int_{E} d^{2} \mathbf{s} / \delta\left(\mathbf{s}-\mathbf{s}^{\prime}\right) \mathbf{V}_{n}^{*}\left(\mathbf{s}, z_{b}\right) \\
& \cdot \mathbf{U}_{K}\left(\mathbf{s}^{\prime}, z_{b}\right) \int_{z_{b}-\epsilon}^{\infty} d z^{\prime} \delta\left(z_{b}+\epsilon-z^{\prime}\right) \\
&-\int_{C} d^{2} \mathbf{s} \int_{E} d^{2} \mathbf{s}^{/} \delta\left(\mathbf{s}-\mathbf{s}^{\prime}\right) \mathbf{U}_{K}\left(\mathbf{s}^{\prime}, z_{b}\right) \cdot \partial / \partial z\left[\mathbf{V}_{n}^{*}(\mathbf{s}, z)\right]_{z_{b}} \\
& \int_{z_{b}-\epsilon}^{\infty} d z^{\prime} \int_{z_{0}}^{z_{b}+\epsilon} d z \delta\left(z-z^{\prime}\right) .
\end{aligned}
$$

In equation (162) the first term is non-zero, since the $z$ / integral equals one and we are left with a surface integral over the cavity-external region boundary when the $\delta\left(\mathbf{s}-\mathbf{s}^{\prime}\right)$ factor is integrated out. The second term in equation (162) is zero, the integrals over $z$ and $z^{\prime}$ giving a result proportional to the small quantity $\epsilon$, and as in the $I_{x}$ case the overall integral is zero. Hence we have:

$$
I_{z}=\int_{S} d^{2} \mathbf{s} \mathbf{V}_{n}^{*}\left(\mathbf{s}, z_{b}\right) \cdot \mathbf{U}_{K}\left(\mathbf{s}, z_{b}\right)
$$

and thus

$$
\left[\hat{Q}_{n}, \hat{P}_{K}^{\dagger}\right] \cdot=\left(\hbar / k_{n}\right) \int_{S} d^{2} \mathbf{s} \mathbf{V}_{n}^{*}\left(\mathbf{s}, z_{b}\right) \cdot \mathbf{U}_{K}\left(\mathbf{s}, z_{b}\right)
$$

\subsubsection{Case of $\left[\hat{R}_{n}, \hat{S}_{K}^{\dagger}\right]$}

To obtain the commutation rule for $\left[\hat{R}_{n}, \hat{S}_{K}^{\dagger}\right]$, we first obtain the result for $\left[\hat{R}_{n}^{\dagger}, \hat{S}_{K}\right]$ by multiplying each side of equation (154) by $U_{n}(\mathbf{R}) V_{K}^{\beta *}\left(\mathbf{R}^{\prime}\right)$, integrating $\mathbf{R}, \mathbf{R}^{\prime}$ over the cavity and external regions respectively, then summing over $\beta$. The treatment then involves the same steps as in the case of $\left[\hat{Q}_{n}, \hat{P}_{K}^{\dagger}\right]$ except for the replacement of $V_{n}^{*}$ by $U_{n}$ and $U_{K}^{\beta}$ by $V_{K}^{\beta *}$ together with the presence of a negative sign resulting from the original equation (154) itself. This leads to:

$$
\left[\hat{R}_{n}^{\dagger}, \hat{S}_{K}\right]=-\left(\hbar / k_{n}\right) \int_{S} d^{2} \mathbf{s} \mathbf{U}_{n}\left(\mathbf{s}, z_{b}\right) \cdot \mathbf{V}_{K}^{*}\left(\mathbf{s}, z_{b}\right)
$$


so after taking the adjoint of both sides of this result we have finally:

$$
\left[\hat{R}_{n}, \hat{S}_{K}^{\dagger}\right]=\left(\hbar / k_{n}\right) \int_{S} d^{2} \mathbf{s} \mathbf{U}_{n}^{*}\left(\mathbf{s}, z_{b}\right) \cdot \mathbf{V}_{K}\left(\mathbf{s}, z_{b}\right) .
$$

\subsubsection{Cases of $\left[\hat{Q}_{n}, \hat{S}_{K}^{\dagger}\right]$ and $\left[\hat{R}_{n}, \hat{P}_{K}^{\dagger}\right]$}

To obtain the commutation rules for $\left[\hat{Q}_{n}, \hat{S}_{K}^{\dagger}\right]$ and $\left[\hat{R}_{n}, \hat{P}_{K}^{\dagger}\right]$ we use equations (21) to write $\hat{Q}_{n}$ as a linear combination of the $\hat{R}_{m}$ and $\hat{R}_{n}$ as a linear combination of the $\hat{Q}_{m}$. This involves the transformation matrices $\mathbf{D}_{n m}$ and $\mathbf{C}_{n m}$ respectively. We then use the previously derived results in equations (166, 164) for the commutators $\left[\hat{R}_{m}, \hat{S}_{K}^{\dagger}\right]$ and $\left[\hat{Q}_{m}, \hat{P}_{K}^{\dagger}\right]$. The $\left(\hbar / k_{m}\right)$ terms in the sums over $m$ can be replaced by $\left(\hbar / k_{n}\right)$ since the transformation matrices are zero unless $k_{n}=k_{m}$. We then use equations (14) and the Hermiteancy properties of $\mathbf{D}_{n m}$ and $\mathbf{C}_{n m}$ to perform the sum over $m$, and this leads to the NHM functions $\mathbf{U}_{m}^{*}$ and $\mathbf{V}_{m}^{*}$ being replaced by $\mathbf{V}_{n}^{*}$ and $\mathbf{U}_{n}^{*}$ respectively. This gives the results:

$$
\begin{aligned}
& {\left[\hat{Q}_{n}, \hat{S}_{K}^{\dagger}\right]=\left(\hbar / k_{n}\right) \int_{S} d^{2} \mathbf{s} \mathbf{V}_{n}^{*}\left(\mathbf{s}, z_{b}\right) \cdot \mathbf{V}_{K}\left(\mathbf{s}, z_{b}\right)} \\
& {\left[\hat{R}_{n}, \hat{P}_{K}^{\dagger}\right]=\left(\hbar / k_{n}\right) \int_{S} d^{2} \mathbf{s} \mathbf{U}_{n}^{*}\left(\mathbf{s}, z_{b}\right) \cdot \mathbf{U}_{K}\left(\mathbf{s}, z_{b}\right) .}
\end{aligned}
$$

\subsubsection{Cases of $\left[\hat{Q}_{n}, \hat{P}_{K}\right],\left[\hat{R}_{n}, \hat{S}_{K}\right],\left[\hat{Q}_{n}, \hat{S}_{K}\right],\left[\hat{R}_{n}, \hat{P}_{K}\right]$ and their ad- joints}

These commutators involve either two or zero adjoints and we conclude they may be taken as equal to zero. Starting from equation (154) we could multiply each side by $V_{n}^{*}(\mathbf{R}) V_{K}^{\beta *}\left(\mathbf{R}^{\prime}\right)$, integrate $\mathbf{R}, \mathbf{R}^{\prime}$ over the cavity and external regions respectively, then sum over $\beta$ and follow similar steps as for $\left[\hat{Q}_{n}, \hat{P}_{K}^{\dagger}\right]$ to obtain a surface integral expression for $\left[\hat{Q}_{n}, \hat{S}_{K}\right]$. The surface integral would involve the scalar product $\mathbf{V}_{n}^{*}\left(\mathbf{s}, z_{b}\right) \cdot \mathbf{V}_{K}^{*}\left(\mathbf{s}, z_{b}\right)$ with both factors conjugated. The cavity-external boundary is never really planar, so the exponential factors associated with $\mathbf{V}_{n}^{*}\left(\mathbf{s}, z_{b}\right) \cdot \mathbf{V}_{K}^{*}\left(\mathbf{s}, z_{b}\right)$ would average out to zero. On this basis we neglect commutators such as $\left[\hat{Q}_{n}, \hat{S}_{K}\right],\left[\hat{R}_{n}, \hat{S}_{K}\right],\left[\hat{Q}_{n}, \hat{S}_{K}\right],\left[\hat{R}_{n}, \hat{P}_{K}\right]$ and their adjoints. Alternatively, using equations (18) for the cavity NHM generalised coordinates and those analogous to equation (48) for the external NHM generalised momenta, we see that such commutators would be linear combinations of true mode commutators $\left[\hat{q}_{k}, \hat{p}_{l}\right],\left[\hat{q}_{k}^{\dagger}, \hat{p}_{l}^{\dagger}\right]$ that are all zero.

\subsection{Cases of cavity NHM momenta and external NHM coordinates}

The commutation rules between cavity NHM momenta and external region coordinates are found by substituting for $\hat{\mathbf{D}}(\mathbf{R})$ and $\hat{\mathbf{B}}\left(\mathbf{R}^{\prime}\right)$ from equations (151, 153 into equation (149), which gives: 


$$
\begin{aligned}
i \hbar \sum_{\gamma} \varepsilon_{\alpha \beta \gamma} \partial / \partial X_{\gamma} \delta\left(\mathbf{R}-\mathbf{R}^{\prime}\right)=\sum_{m(\alpha)} \sum_{L}\left\{U_{m}(\mathbf{R})\left\{\boldsymbol{\nabla} \times \mathbf{U}_{L}\left(\mathbf{R}^{\prime}\right)\right\}^{\beta}\left[\hat{S}_{m}, \hat{Q}_{L}\right]\right. \\
+U_{m}(\mathbf{R})\left\{\boldsymbol{\nabla} \times \mathbf{V}_{L}\left(\mathbf{R}^{\prime}\right)\right\}^{\beta *}\left[\hat{S}_{m}, \hat{R}_{L}^{\dagger}\right] \\
+V_{m}^{*}(\mathbf{R})\left\{\boldsymbol{\nabla} \times \mathbf{U}_{L}\left(\mathbf{R}^{\prime}\right)\right\}^{\beta}\left[\hat{P}_{m}^{\dagger}, \hat{Q}_{L}\right] \\
\left.+V_{m}^{*}(\mathbf{R})\left\{\boldsymbol{\nabla} \times \mathbf{V}_{L}\left(\mathbf{R}^{\prime}\right)\right\}^{\beta *}\left[\hat{P}_{m}^{\dagger}, \hat{R}_{L}^{\dagger}\right]\right\} .
\end{aligned}
$$

On the right side the sum over $m$ only involves terms with $\hat{\boldsymbol{\alpha}}_{m}=\hat{\boldsymbol{\alpha}}$.

\subsubsection{Case of $\left[\hat{S}_{n}, \hat{R}_{K}^{\dagger}\right]$}

To obtain the commutation rule for $\left[\hat{S}_{n}, \hat{R}_{K}^{\dagger}\right]$ we multiply each side of equation (169) by $V_{n}^{*}(\mathbf{R})\left\{\boldsymbol{\nabla} \times \mathbf{U}_{K}\left(\mathbf{R}^{\prime}\right)\right\}^{\beta}$, integrate $\mathbf{R}, \mathbf{R}^{\prime}$ over the cavity and external regions respectively, then sum over $\beta$. Using the biorthogonality results in equation (10) and the results in equations (39, 40) for the magnetic energy term and (as usual) neglecting integrals where the complex conjugation occurs either zero or two times we find that:

$$
\begin{gathered}
k_{K}^{2}\left[\hat{S}_{n}, \hat{R}_{K}^{\dagger}\right]=i \hbar \sum_{\gamma} \varepsilon_{\alpha \beta \gamma} \int_{C} d^{3} \mathbf{R} \int_{E} d^{3} \mathbf{R}^{/} V_{n}^{*}(\mathbf{R})\left\{\boldsymbol{\nabla} \times \mathbf{U}_{K}\left(\mathbf{R}^{\prime}\right)\right\}^{\beta} \\
. \partial / \partial X_{\gamma} \delta\left(\mathbf{R}-\mathbf{R}^{\prime}\right)
\end{gathered}
$$

where $\boldsymbol{\alpha}_{n}=\hat{\boldsymbol{\alpha}}$. Since $\boldsymbol{\alpha}_{n}=\hat{\mathbf{x}}$ or $\boldsymbol{\alpha}_{n}=\hat{\mathbf{y}}$ are the only two possibilities we get the two equations:

$$
\begin{aligned}
k_{K}^{2}\left[\hat{S}_{n}, \hat{R}_{K}^{\dagger}\right]= & i \hbar \int_{C} d^{3} \mathbf{R} \int_{E} d^{3} \mathbf{R}^{\prime} V_{n}^{*}(\mathbf{R})\left\{\boldsymbol{\nabla} \times \mathbf{U}_{K}\left(\mathbf{R}^{\prime}\right)\right\}^{y} \partial / \partial z \delta\left(\mathbf{R}-\mathbf{R}^{\prime}\right) \\
& -i \hbar \int_{C} d^{3} \mathbf{R} \int_{E} d^{3} \mathbf{R}^{\prime} V_{n}^{*}(\mathbf{R})\left\{\boldsymbol{\nabla} \times \mathbf{U}_{K}\left(\mathbf{R}^{\prime}\right)\right\}^{z} \partial / \partial y \delta\left(\mathbf{R}-\mathbf{R}^{\prime}\right)
\end{aligned}
$$

for $\boldsymbol{\alpha}_{n}=\hat{\mathbf{x}}$ and

$$
\begin{aligned}
k_{K}^{2}\left[\hat{S}_{n}, \hat{R}_{K}^{\dagger}\right]= & -i \hbar \int_{C} d^{3} \mathbf{R} \int_{E} d^{3} \mathbf{R}^{/} V_{n}^{*}(\mathbf{R})\left\{\boldsymbol{\nabla} \times \mathbf{U}_{K}\left(\mathbf{R}^{\prime}\right)\right\}^{x} \partial / \partial z \delta\left(\mathbf{R}-\mathbf{R}^{\prime}\right) \\
& +i \hbar \int_{C} d^{3} \mathbf{R} \int_{E} d^{3} \mathbf{R}^{/} V_{n}^{*}(\mathbf{R})\left\{\boldsymbol{\nabla} \times \mathbf{U}_{K}\left(\mathbf{R}^{\prime}\right)\right\}^{z} \partial / \partial x \delta\left(\mathbf{R}-\mathbf{R}^{\prime}\right)
\end{aligned}
$$

for $\boldsymbol{\alpha}_{n}=\hat{\mathbf{y}}$.

As previously, the integrals involving $\partial / \partial x$ or $\partial / \partial y$ give zero, so only the integrals involving $\partial / \partial z$ remain. Following the same treatment for the latter 
results in expressions involving surface integrals:

$$
k_{K}^{2}\left[\hat{S}_{n}, \hat{R}_{K}^{\dagger}\right]=i \hbar \int_{S} d^{2} \mathbf{s} V_{n}^{*}\left(\mathbf{s}, z_{b}\right)\left\{\nabla \times \mathbf{U}_{K}\left(\mathbf{s}, z_{b}\right)\right\}^{y}
$$

for $\boldsymbol{\alpha}_{n}=\hat{\mathbf{x}}$ and

$$
k_{K}^{2}\left[\hat{S}_{n}, \hat{R}_{K}^{\dagger}\right]=-i \hbar \int_{S} d^{2} \mathbf{s} V_{n}^{*}\left(\mathbf{s}, z_{b}\right)\left\{\nabla \times \mathbf{U}_{K}\left(\mathbf{s}, z_{b}\right)\right\}^{x}
$$

or $\boldsymbol{\alpha}_{n}=\hat{\mathbf{y}}$. These results may be summarised in a single formula:

$$
\left[\hat{S}_{n}, \hat{R}_{K}^{\dagger}\right]=\left(i \hbar / k_{K}^{2}\right) \int_{S} d^{2} \mathbf{s} \hat{\mathbf{z}} \cdot \mathbf{V}_{n}^{*}\left(\mathbf{s}, z_{b}\right) \times\left\{\nabla \times \mathbf{U}_{K}\left(\mathbf{s}, z_{b}\right)\right\} .
$$

\subsubsection{Case of $\left[\hat{P}_{n}, \hat{Q}_{K}^{\dagger}\right]$}

To obtain the commutation rule for $\left[\hat{P}_{n}, \hat{Q}_{K}^{\dagger}\right]$, we first obtain the result for $\left[\hat{P}_{n}^{\dagger}, \hat{Q}_{K}\right]$ by multiplying each side of equation (169) by $U_{n}(\mathbf{R})\left\{\boldsymbol{\nabla} \times \mathbf{V}_{K}\left(\mathbf{R}^{\prime}\right)\right\}^{\beta *}$, integrating $\mathbf{R}, \mathbf{R}^{\prime}$ over the cavity and external regions respectively, then summing over $\beta$. The treatment then involves the same steps as in the case of $\left[\hat{S}_{n}, \hat{R}_{K}^{\dagger}\right]$ except for the replacement of $V_{n}^{*}$ by $U_{n}$ and $\left\{\boldsymbol{\nabla} \times \mathbf{U}_{K}\left(\mathbf{R}^{\prime}\right)\right\}^{\beta}$ by $\left\{\boldsymbol{\nabla} \times \mathbf{V}_{K}\left(\mathbf{R}^{/}\right)\right\}^{\beta *}$. This leads to:

$$
\left[\hat{P}_{n}^{\dagger}, \hat{Q}_{K}\right]=\left(i \hbar / k_{K}^{2}\right) \int_{S} d^{2} \mathbf{s} \hat{\mathbf{z}} \cdot \mathbf{U}_{n}\left(\mathbf{s}, z_{b}\right) \times\left\{\nabla \times \mathbf{V}_{K}^{*}\left(\mathbf{s}, z_{b}\right)\right\},
$$

and taking the adjoint of both sides leads to the result:

$$
\left[\hat{P}_{n}, \hat{Q}_{K}^{\dagger}\right]=\left(i \hbar / k_{K}^{2}\right) \int_{S} d^{2} \mathbf{s} \hat{\mathbf{z}} \cdot \mathbf{U}_{n}^{*}\left(\mathbf{s}, z_{b}\right) \times\left\{\nabla \times \mathbf{V}_{K}\left(\mathbf{s}, z_{b}\right)\right\} .
$$

\subsubsection{Cases of $\left[\hat{P}_{n}, \hat{R}_{K}^{\dagger}\right]$ and $\left[\hat{S}_{n}, \hat{Q}_{K}^{\dagger}\right]$}

To obtain the commutation rules for $\left[\hat{P}_{n}, \hat{R}_{K}^{\dagger}\right]$ and $\left[\hat{S}_{n}, \hat{Q}_{K}^{\dagger}\right]$ we use equations (49) to write $\hat{P}_{n}$ as a linear combination of the $\hat{S}_{m}$ and $\hat{S}_{n}$ as a linear combination of the $\hat{P}_{m}$. The same method used for determining $\left[\hat{Q}_{n}, \hat{S}_{K}^{\dagger}\right]$ and $\left[\hat{R}_{n}, \hat{P}_{K}^{\dagger}\right]$ is then followed and we find that:

$$
\begin{aligned}
& {\left[\hat{P}_{n}, \hat{R}_{K}^{\dagger}\right]=\left(i \hbar / k_{K}^{2}\right) \int_{S} d^{2} \mathbf{s} \hat{\mathbf{z}} \cdot \mathbf{U}_{n}^{*}\left(\mathbf{s}, z_{b}\right) \times\left\{\nabla \times \mathbf{U}_{K}\left(\mathbf{s}, z_{b}\right)\right\}} \\
& {\left[\hat{S}_{n}, \hat{Q}_{K}^{\dagger}\right]=\left(i \hbar / k_{K}^{2}\right) \int_{S} d^{2} \mathbf{s} \hat{\mathbf{z}} \cdot \mathbf{V}_{n}^{*}\left(\mathbf{s}, z_{b}\right) \times\left\{\nabla \times \mathbf{V}_{K}\left(\mathbf{s}, z_{b}\right)\right\} .}
\end{aligned}
$$

\subsubsection{Cases of $\left[\hat{P}_{n}, \hat{Q}_{K}\right],\left[\hat{S}_{n}, \hat{R}_{K}\right],\left[\hat{P}_{n}, \hat{R}_{K}\right],\left[\hat{S}_{n}, \hat{Q}_{K}\right]$ and their ad- joints}

These commutators involve either two or zero adjoints and we conclude they may be taken as equal to zero. The details are similar to those for $\left[\hat{Q}_{n}, \hat{P}_{K}\right]$, $\left[\hat{R}_{n}, \hat{S}_{K}\right],\left[\hat{Q}_{n}, \hat{S}_{K}\right],\left[\hat{R}_{n}, \hat{P}_{K}\right]$ and their adjoints. 


\section{References}

[1] Huttner, B. and Barnett, S. M., 1992, Phys. Rev. A46, 4306-4322.

[2] Knoll, L., Vogel, W., and Welsch, D.-G., 1987, Phys. Rev. A36, 3803-3818.

[3] Glauber, R. J., and Lewenstein, M., 1991, Phys. Rev. A43, 467-491.

[4] Dalton, B. J., Guerra, E. S., and Knight, P. L., 1996, Phys. Rev. A54, 2292-2313.

[5] Dalton, B. J., and Babiker, M., 1997, Phys. Rev. A56, 905-911.

[6] Dalton, B. J., Barnett, S. M., and Knight, P. L., 1999, J. Mod. Opt. 46, 1315-1341, ibid 1495-1502.

[7] Dalton, B. J., Barnett, S. M., and Knight, P. L., 1999, J. Mod. Opt. 46, 1559-1577.

[8] Dalton, B. J., and Knight, P. L., 1999, J. Mod. Opt. 46, 1817-1837, ibid 1839-1868.

[9] Brown, S., and Dalton, B. J., 2001, J. Mod. Opt. 48, 639-670.

[10] Brown, S., and Dalton, B. J., 2001, J. Mod. Opt. 48, 597-618.

[11] Siegman, A. E., 1995, App. Phys. B60, 247-257.

[12] Petermann, K., 1979, IEEE J. Quantum Electron. 15, 566-570.

[13] Siegman, A. E., 1989, Phys. Rev. A39, 1253-1263, ibid 1264-1268.

[14] Hamel, W. A., and Woerdman, J. P., 1989, Phys. Rev. A40, 2785-2787.

[15] Rippin, M. A., and New, G. H. C., 1996, J. Mod. Opt. 43, 993-1008.

[16] Deutsch, I. H., Garrison, J. C., and Wright, E. M., 1991, J. Opt. Soc. Amer. B6, 1244-1251.

[17] Goldberg, P., Milonni, P. W., and Sundaram, B., 1991, Phys. Rev. A44, 1969-1985.

[18] Grangier, P., and Poizat, J.-P., 1998, Eur. Phys. D1, 97-104.

[19] Lamprecht, C., and Ritsch, H., 1999, Phys. Rev. Lett. 82, 3787-3790.

[20] Bardroff, P. J., and Stenholm, S., 1999, Phys. Rev. A60, 2529-2533.

[21] Dutra, S. M., and Nienhuis, G., 2000, Phys. Rev. A62, 063805 (1-13).

[22] Lax, M., Louisell, W. H., and McKnight, W. B., 1975, Phys. Rev. A11, 1365-1370. 
[23] Milonni, P. W., and Eberly, J. H., 1988, Lasers (New York: Wiley). Chapter 14 .

[24] Siegman, A. E., 1986, Lasers (Mill Valley, CA: University Science Books). Chapter 16.

\section{$7 \quad$ Figure captions}

Figure 1. Unstable optical resonator system consisting of two confocal mirrors. The dashed line indicates the boundary between the cavity and the external regions. The left hand mirror is assumed to be large, so that light does not travel past it and is confined to the cavity and external regions shown.

Figure 2. Ranges of $z$ integration for cavity and external regions used to determine cavity NHM/external NHM commutation rules. 


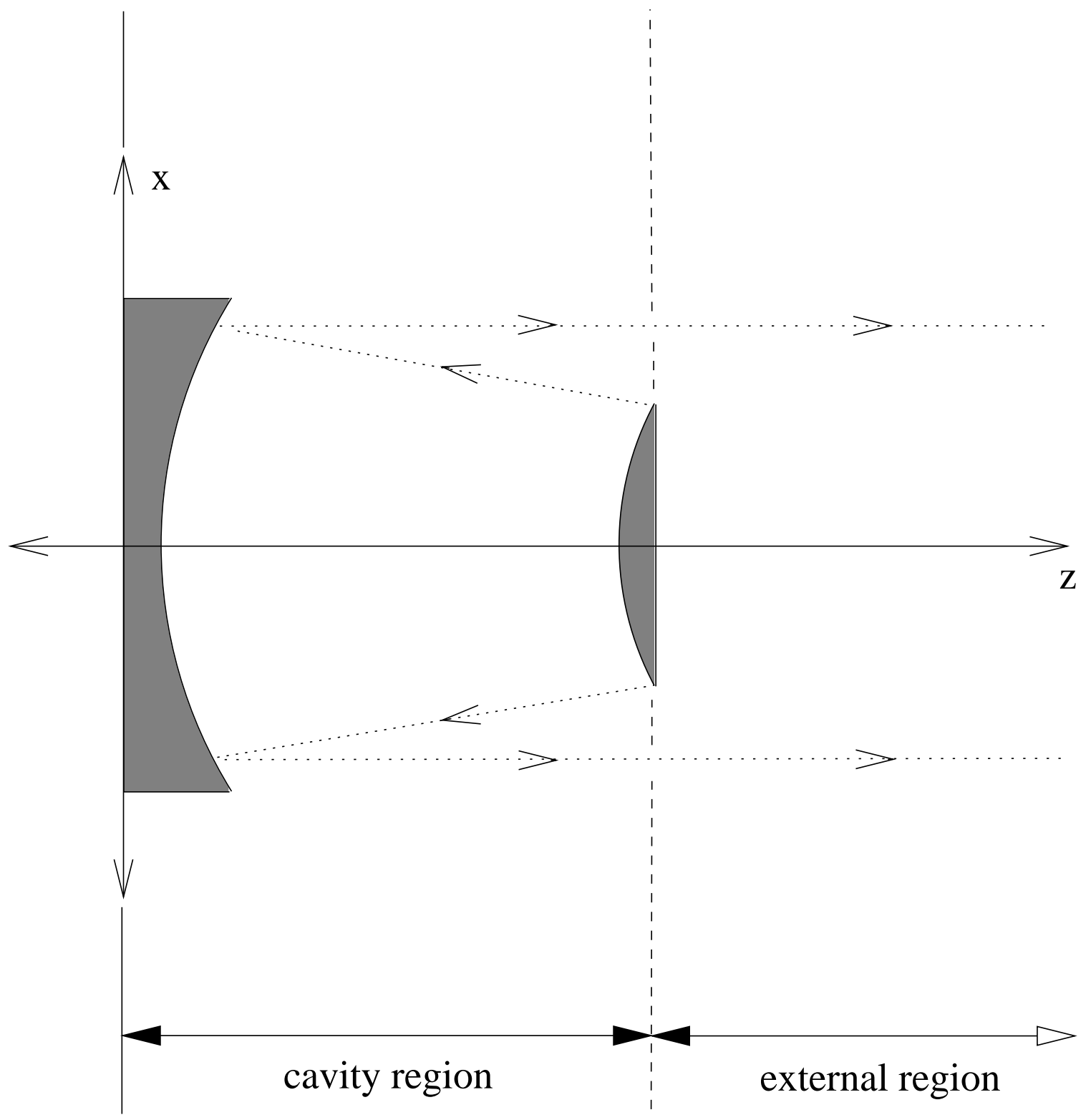




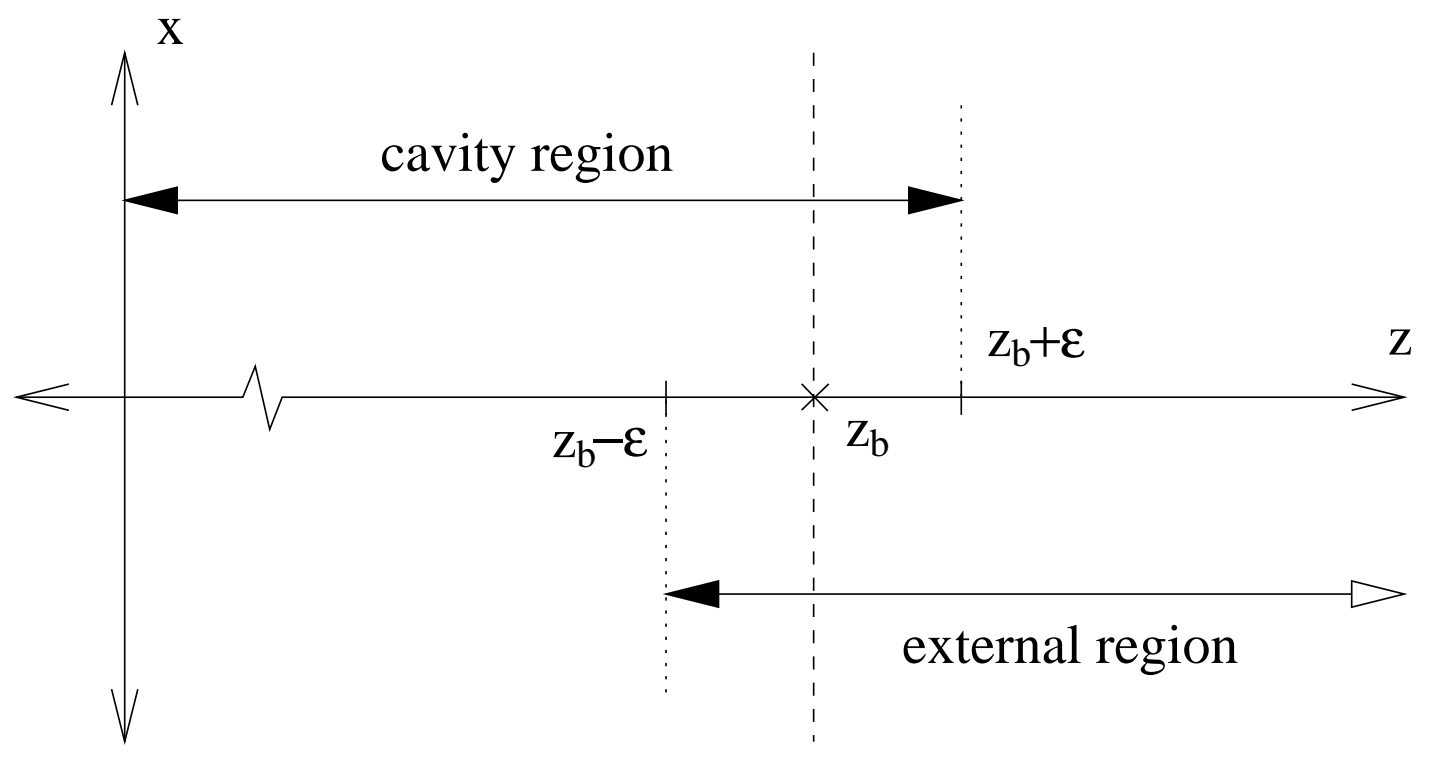

